\title{
Equivalence Checking of Static Affine Programs using Widening to Handle Recurrences
}

Sven Verdoolaege Gerda Janssens Maurice Bruynooghe

\author{
Katholieke Universiteit Leuven, Belgium
}

June 30, 2009 


\section{Equivalence Checking Example}

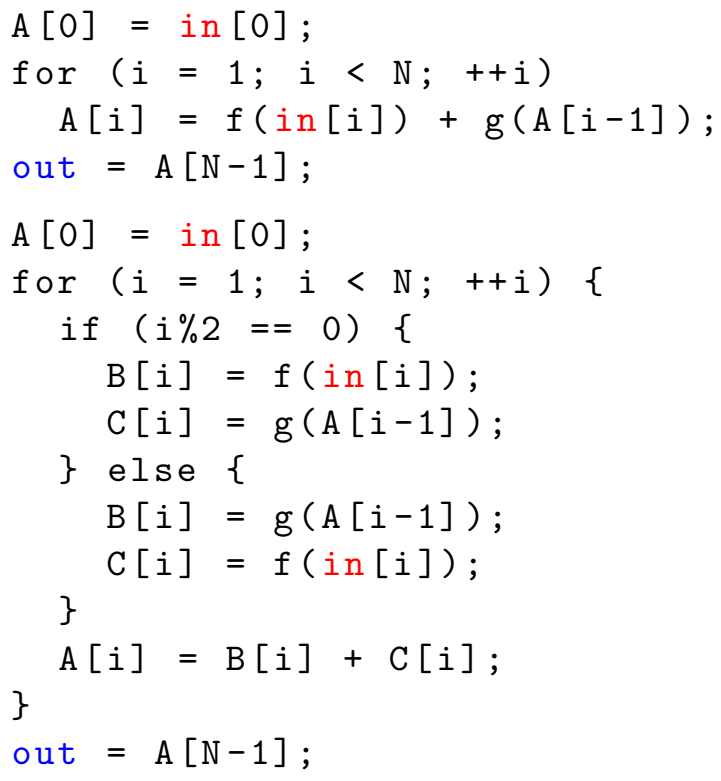




\section{Equivalence Checking Example}

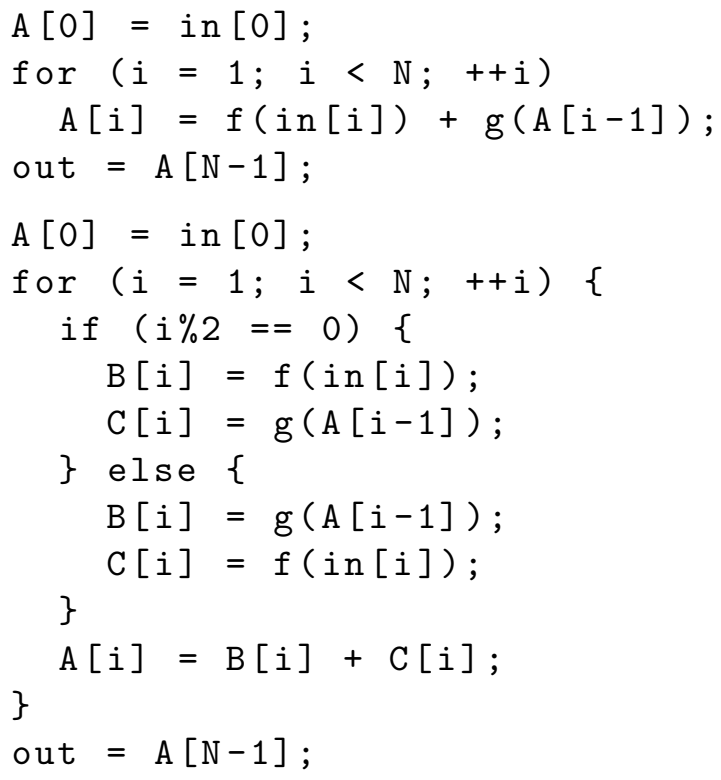

Are the two programs on the left equivalent?

$\Rightarrow$ Same output when given same input

Assumptions

- no pointers

- no recursion

- functions called are pure

- static control flow

- quasi-affine loop bounds

- quasi-affine conditions

- quasi-affine index expressions

Main challenges:

- Recurrences

- Transformations exploiting commutativity 


\section{Equivalence Checking Example}

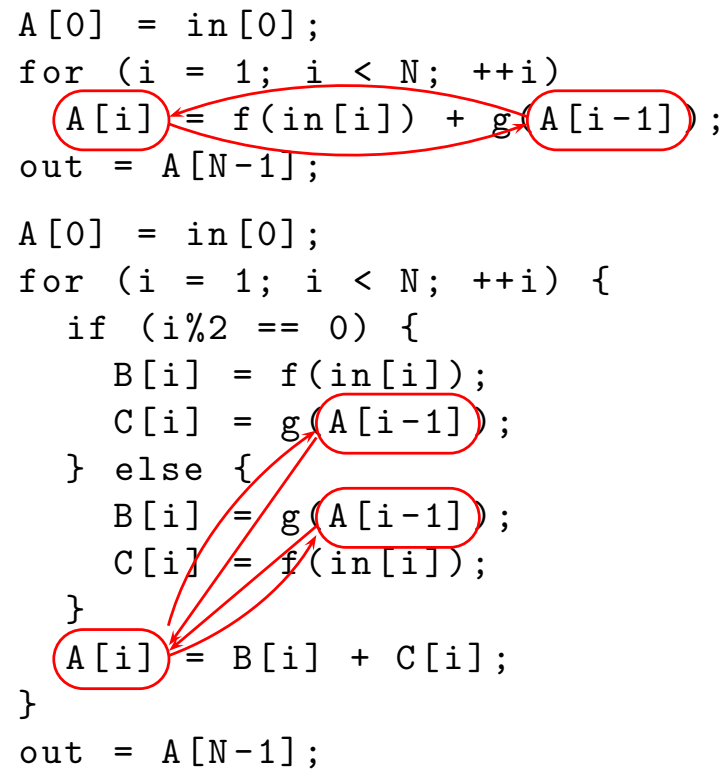

Are the two programs on the left equivalent?

$\Rightarrow$ Same output when given same input

Assumptions

- no pointers

- no recursion

- functions called are pure

- static control flow

- quasi-affine loop bounds

- quasi-affine conditions

- quasi-affine index expressions

Main challenges:

- Recurrences

- Transformations exploiting commutativity 


\section{Equivalence Checking Example}

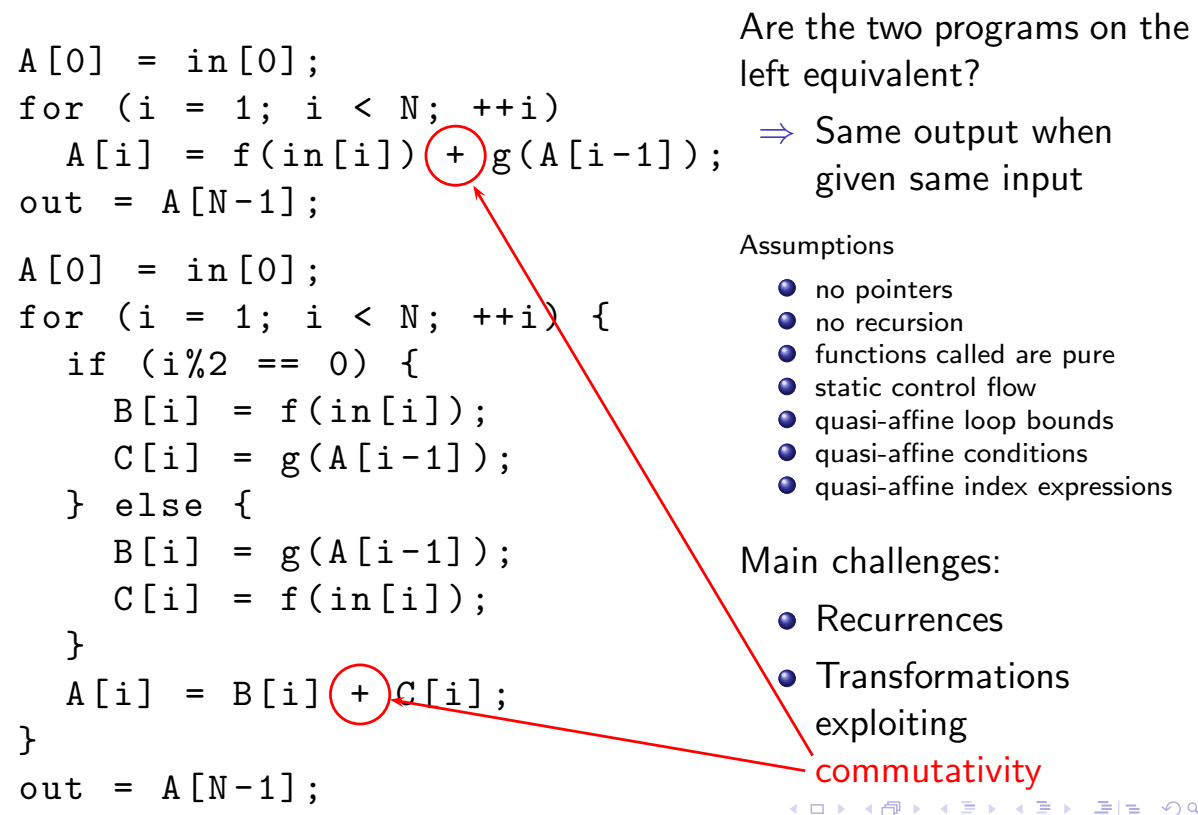

Are the two programs on the left equivalent?

$\Rightarrow$ Same output when given same input

Assumptions

- no pointers

- no recursion

- functions called are pure

- static control flow

- quasi-affine loop bounds

- quasi-affine conditions

- quasi-affine index expressions 


\section{Context}

Multi-media applications for embedded systems

- Manipulation of large multi-dimensional arrays

- Relatively simple control flow, mainly loops

- Strict timing constraints

Transformation are applied

- mainly loop transformations and data transformations

- to improve time and energy efficiency

- (semi)automatically or manually

Question:

Is the transformed program (input-output) equivalent to the original?

$\Rightarrow$ equivalence checking should be fully automated 


\section{Outline}

(1) Motivation

(2) Related Work

(3) Our Approach

- Dependence Graphs

- Propagation

- Commutative Operations

- Associative Operations

- Recurrences

(4) Implementation

(5) Experiments

- CLooG

- Results

(6) Conclusion 


\section{Related Work}

Many approaches have been proposed for equivalence checking, e.g.,

- Translation validation

$\Rightarrow$ requires hints from the compiler

$\Rightarrow$ not available for manual transformations

- General theorem provers

$\Rightarrow$ require user interaction, e.g., to set up induction hypothesis

$\Rightarrow$ not fully automated 


\section{Related Work}

Many approaches have been proposed for equivalence checking, e.g.,

- Translation validation

$\Rightarrow$ requires hints from the compiler

$\Rightarrow$ not available for manual transformations

- General theorem provers

$\Rightarrow$ require user interaction, e.g., to set up induction hypothesis

$\Rightarrow$ not fully automated

Other approaches for static affine programs

- Barthou et al. (2002)

$\Rightarrow$ no support for commutative operations

- Shashidhar et al. (2005)

$\Rightarrow$ limited support for commutative operations

$\Rightarrow$ broken support for recurrences

$\Rightarrow$ Both rely on transitive closure operation for handling recurrences

$\Rightarrow$ only uniform recurrences 


\section{Basic Proof Strategy}

Prove output arrays are equal given that input arrays are equal

( Compute weakest precondition for equal output

$\Rightarrow$ propagate correspondences between values computed in both programs

(2) Check which part is covered by equal input

(3) Propagate results back to output

$\Rightarrow$ which elements of the output arrays are equal

$\Rightarrow$ recurrence handling

$\Rightarrow$ commutativity handling

All functions are assumed to be pure and are treated as black-boxes

$\Rightarrow$ results of function calls equal iff all arguments are equal

Equivalence checking performed on dependence graph abstraction 


\section{Dependence Graphs}

Dependence graph contains the following information: for each value computed in the program, on which values does it depend?

Each computed value is represented by

- function call/operation used to compute value

- iteration vector of the enclosing loops

$\Rightarrow$ complete abstraction of where/how values are stored in memory 


\section{Dependence Graphs}

Dependence graph contains the following information: for each value computed in the program, on which values does it depend?

Each computed value is represented by

- function call/operation used to compute value

- iteration vector of the enclosing loops

$\Rightarrow$ complete abstraction of where/how values are stored in memory

\section{Dependence graph}

- vertices: "computations"

- a (static) function call or operation

- iteration domain $D$ : set of iterations of enclosing loops

- edges: dependences

- argument position $p$

- dependence relation $M$ : maps use to definition

Dependences are obtained through exact dataflow analysis 


\section{Dependence Graphs: Trivial Example}

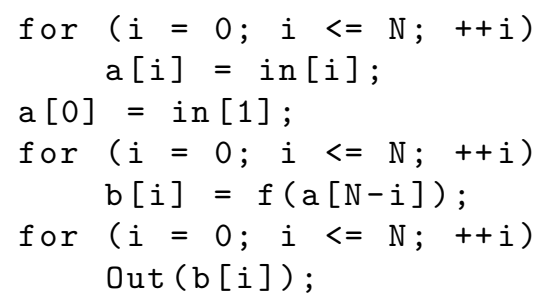

\section{Dependence graph}

- vertices: "computations"

- a (static) function call or operation

- iteration domain $D$ : set of iterations of enclosing loops

- edges: dependences

- argument position $p$

- dependence relation $M$ : maps use to definition

Dependences are obtained through exact dataflow analysis 


\section{Dependence Graphs: Trivial Example}

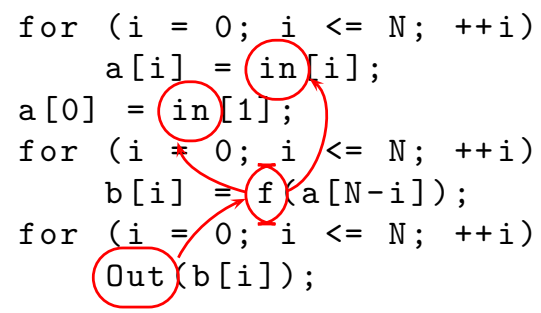

\section{Dependence graph}

- vertices: "computations"

- a (static) function call or operation

- iteration domain $D$ : set of iterations of enclosing loops

- edges: dependences

- argument position $p$

- dependence relation $M$ : maps use to definition

Dependences are obtained through exact dataflow analysis 


\section{Dependence Graphs: Trivial Example}

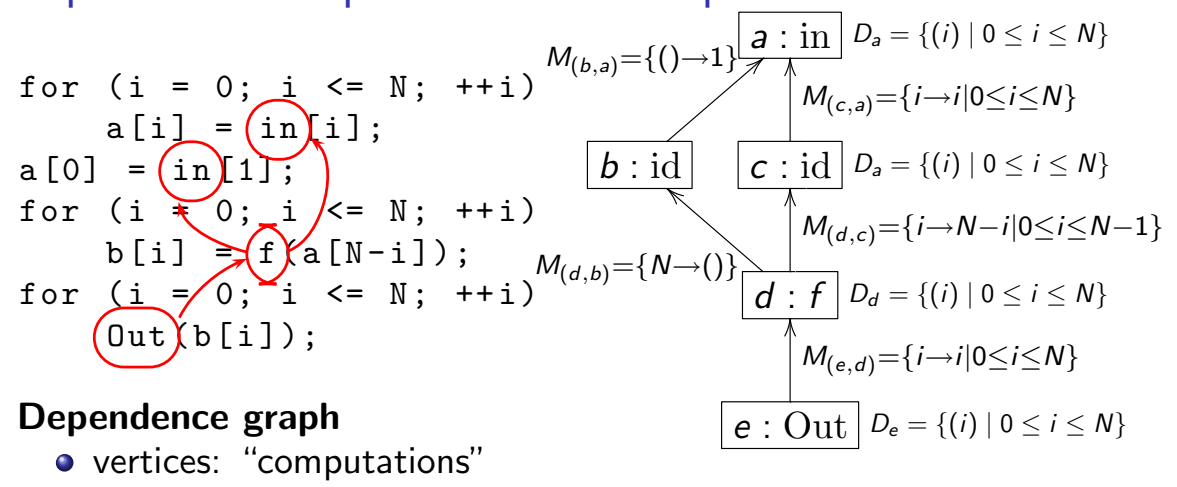

- a (static) function call or operation

- iteration domain $D$ : set of iterations of enclosing loops

- edges: dependences

- argument position $p$

- dependence relation $M$ : maps use to definition

Dependences are obtained through exact dataflow analysis 


\section{Dependence Graphs: Trivial Example}

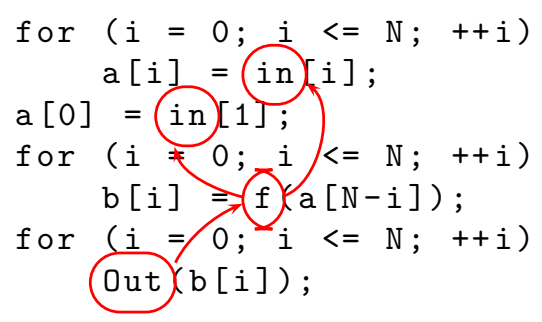

\section{Dependence graph}

- vertices: "computations"

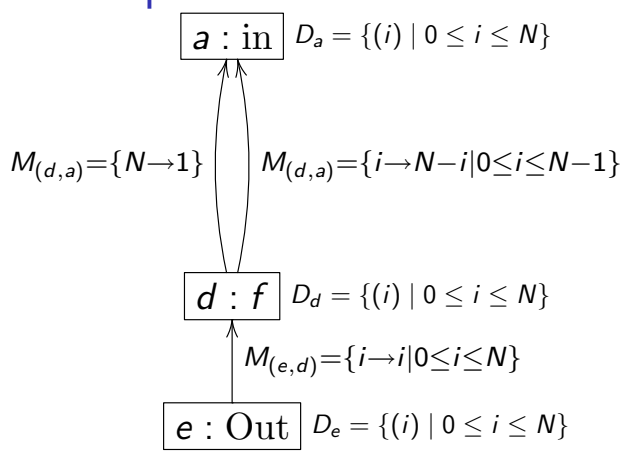

- a (static) function call or operation

- iteration domain $D$ : set of iterations of enclosing loops

- edges: dependences

- argument position $p$

- dependence relation $M$ : maps use to definition

Dependences are obtained through exact dataflow analysis 


\section{Dependence Graphs: Trivial Example}

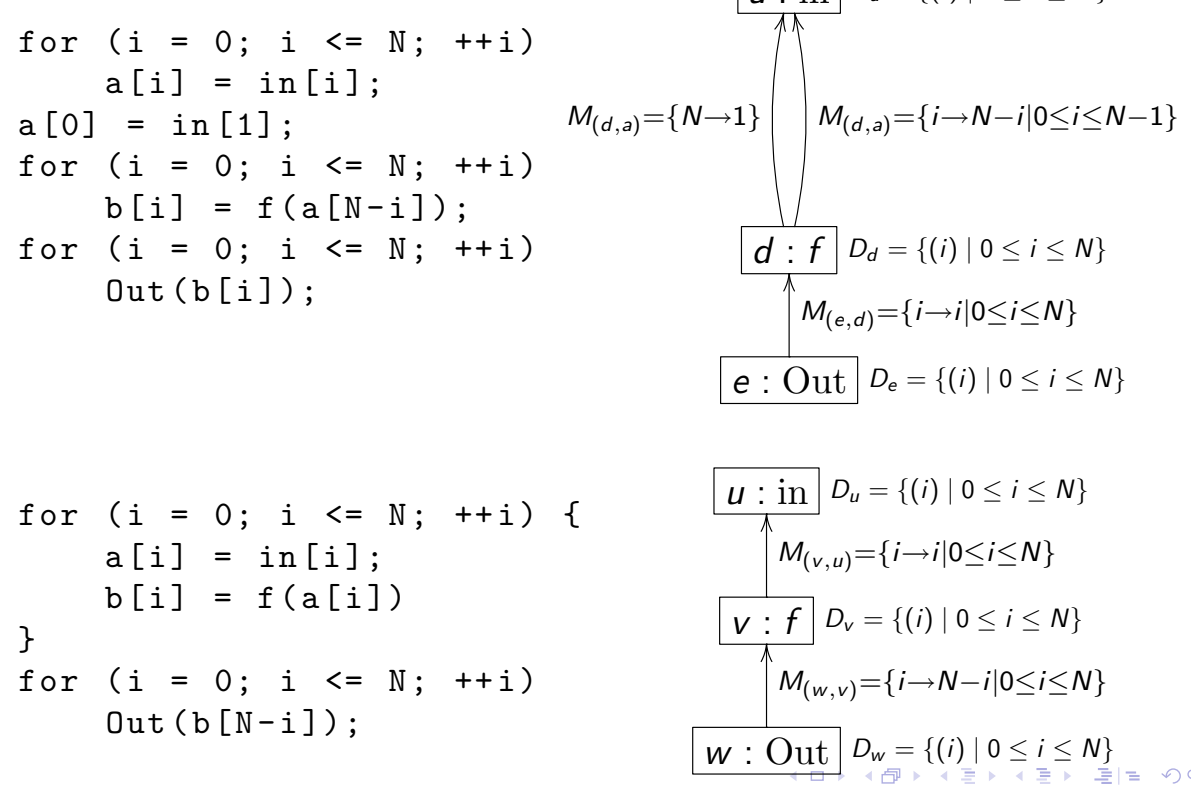




\section{Propagation of Correspondences}

$$
\begin{gathered}
a: \operatorname{in} D_{a}=\{(i) \mid 0 \leq i \leq N\} \\
M_{(d, a)}^{\prime}=\{N \rightarrow 1\} M_{(d, a)}=\{i \rightarrow N-i \mid 0 \leq i \leq N-1\} \\
\frac{d: f D_{b}=\{(i) \mid 0 \leq i \leq N\}}{\uparrow M_{(e, d)}=\{i \rightarrow i \mid 0 \leq i \leq N\}} \\
\text { e : Out } D_{e}=\{(i) \mid 0 \leq i \leq N\}
\end{gathered}
$$

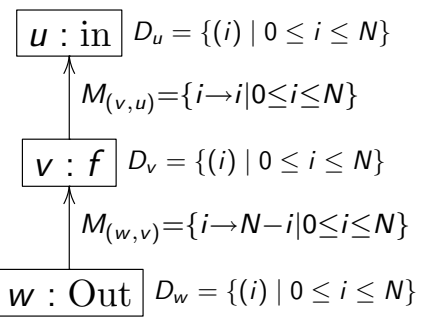




\section{Propagation of Correspondences}

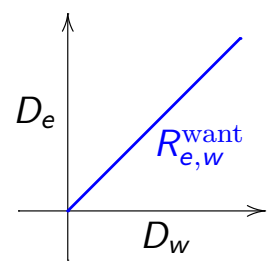

Initial correspondence: $R_{e, w}^{\text {want }}=\{(i, i) \mid 0 \leq i \leq N\}$

$$
\begin{gathered}
a: \text { in } D_{a}=\{(i) \mid 0 \leq i \leq N\} \\
M_{(d, a)}^{\prime}=\{N \rightarrow 1\} M_{(d, a)}=\{i \rightarrow N-i \mid 0 \leq i \leq N-1\} \\
d: f D_{b}=\{(i) \mid 0 \leq i \leq N\} \\
\text { a } M_{(e, d)}=\{i \rightarrow i \mid 0 \leq i \leq N\} \\
e: \text { Out } D_{e}=\{(i) \mid 0 \leq i \leq N\}
\end{gathered}
$$

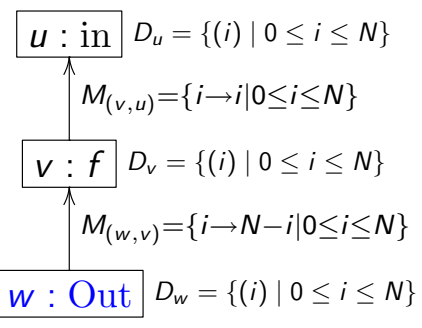




\section{Propagation of Correspondences}

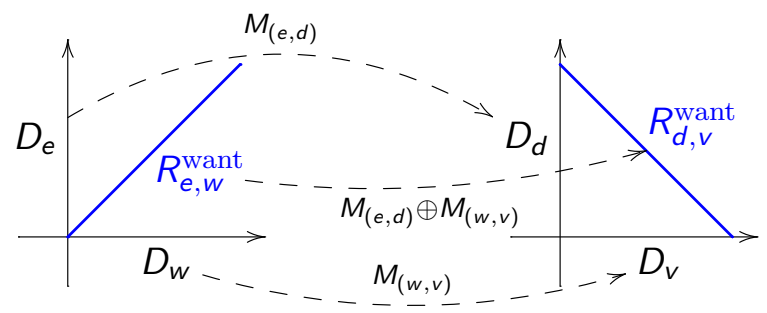

Initial correspondence: $R_{e, w}^{\text {want }}=\{(i, i) \mid 0 \leq i \leq N\}$

Propagation: $R_{d, v}^{\text {want }}=\left(M_{(e, d)} \oplus M_{(w, v)}\right) R_{e, w}^{\text {want }}$

$$
\begin{gathered}
a: \text { in } D_{a}=\{(i) \mid 0 \leq i \leq N\} \\
M_{(d, a)}^{\prime}=\{N \rightarrow 1\} M_{(d, a)}=\{i \rightarrow N-i \mid 0 \leq i \leq N-1\} \\
\frac{d: f D_{b}=\{(i) \mid 0 \leq i \leq N\}}{\uparrow M_{(e, d)}}=\{i \rightarrow i \mid 0 \leq i \leq N\} \\
e: \text { Out } D_{e}=\{(i) \mid 0 \leq i \leq N\}
\end{gathered}
$$

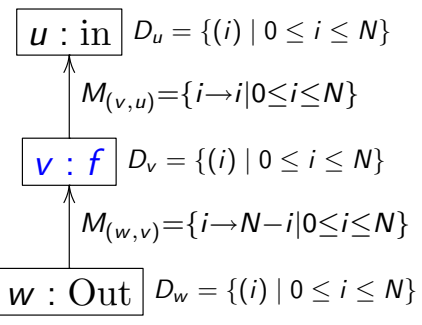




\section{Propagation of Correspondences}

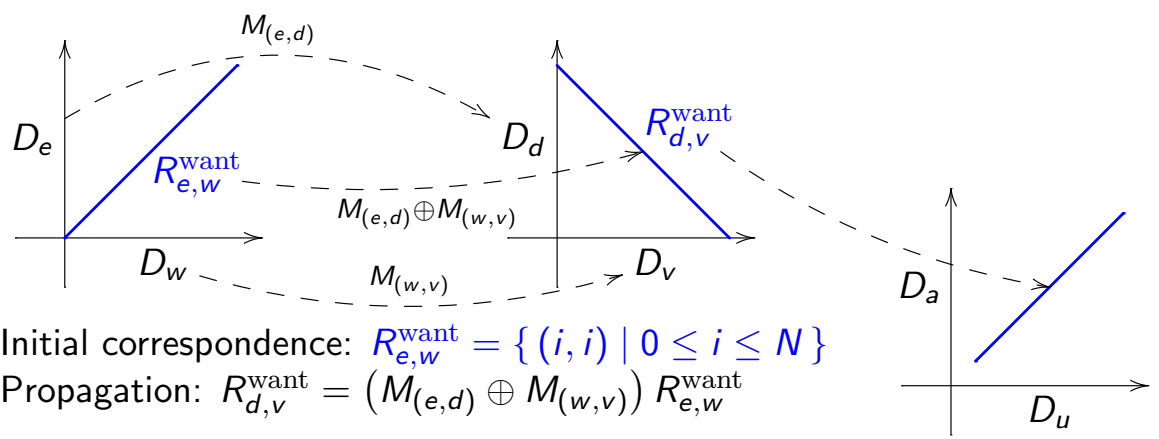

$$
\begin{gathered}
a: \text { in } D_{a}=\{(i) \mid 0 \leq i \leq N\} \\
M_{(d, a)}^{\prime}=\{N \rightarrow 1\} M_{(d, a)}=\{i \rightarrow N-i \mid 0 \leq i \leq N-1\} \\
\frac{d: f D_{b}=\{(i) \mid 0 \leq i \leq N\}}{\uparrow M_{(e, d)}}=\{i \rightarrow i \mid 0 \leq i \leq N\} \\
e: \text { Out } D_{e}=\{(i) \mid 0 \leq i \leq N\}
\end{gathered}
$$

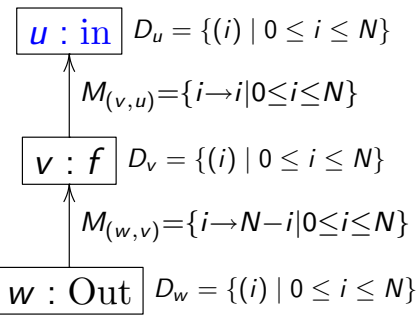




\section{Propagation of Correspondences}

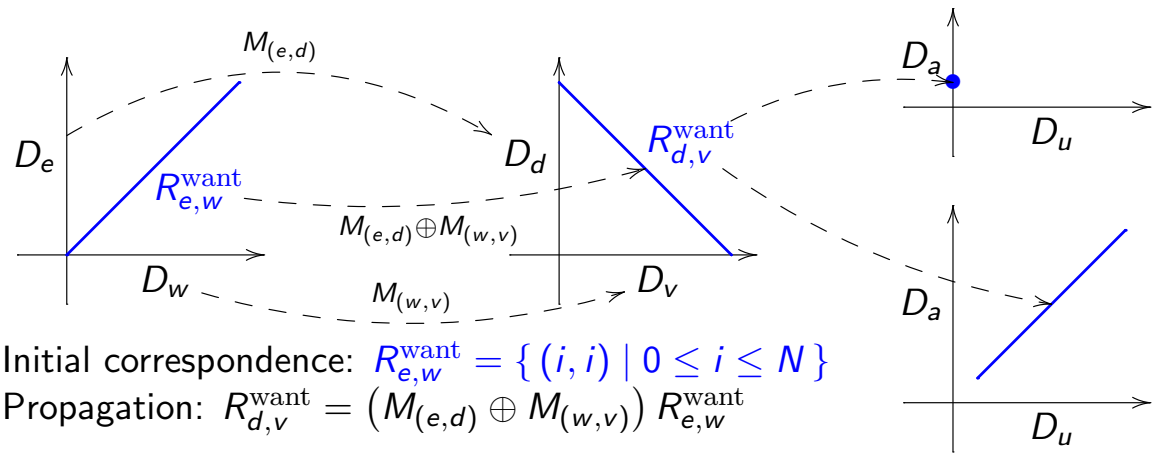

$$
\begin{gathered}
a: \text { in } D_{a}=\{(i) \mid 0 \leq i \leq N\} \\
M_{(d, a)}^{\prime}=\{N \rightarrow 1\} M_{(d, a)}=\{i \rightarrow N-i \mid 0 \leq i \leq N-1\} \\
d: f D_{b}=\{(i) \mid 0 \leq i \leq N\} \\
\text { i } M_{(e, d)}=\{i \rightarrow i \mid 0 \leq i \leq N\} \\
e: \text { Out } D_{e}=\{(i) \mid 0 \leq i \leq N\}
\end{gathered}
$$

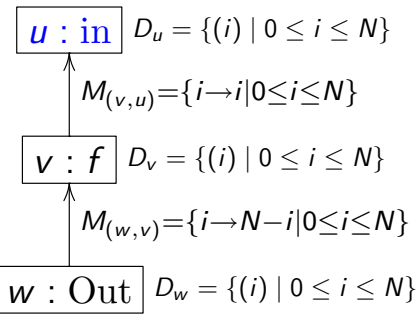




\section{Propagation of Correspondences}

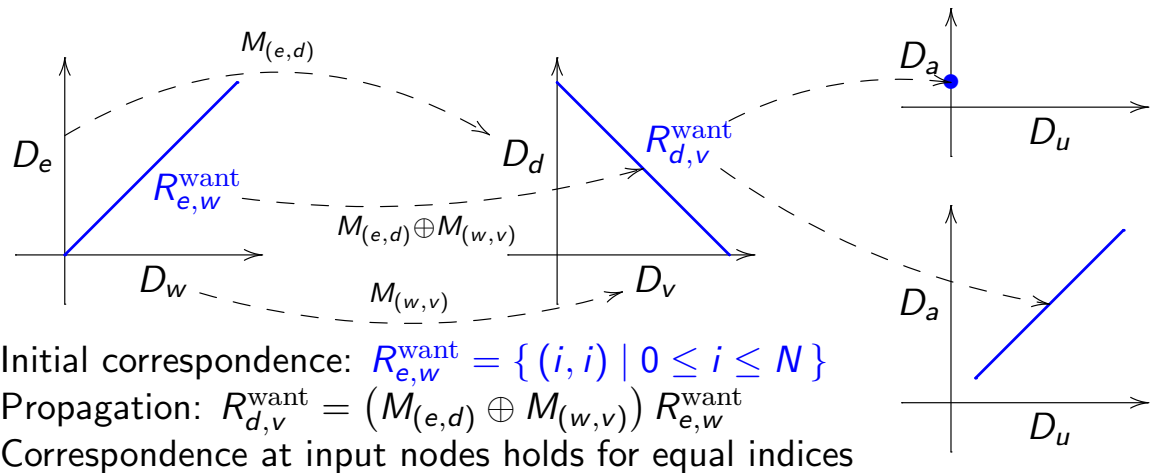

$\Rightarrow R^{\text {got }}=R^{\text {want }} \cap\{(i, i)\}$

$\Rightarrow R^{\text {lost }}=R^{\text {want }} \backslash\{(i, i)\}$ 


\section{Propagation of Correspondences}

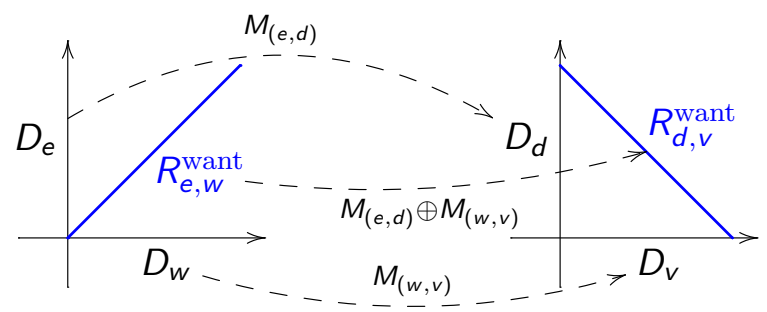

Initial correspondence: $R_{e, w}^{\text {want }}=\{(i, i) \mid 0 \leq i \leq N\}$

Propagation: $R_{d, v}^{\text {want }}=\left(M_{(e, d)} \oplus M_{(w, v)}\right) R_{e, w}^{\text {want }}$

Correspondence at input nodes holds for equal indices

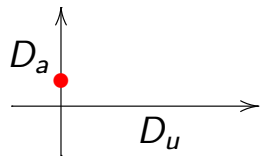

$\Rightarrow R^{\text {got }}=R^{\text {want }} \cap\{(i, i)\}$

$\Rightarrow R^{\text {lost }}=R^{\text {want }} \backslash\{(i, i)\}$ 


\section{Propagation of Correspondences}

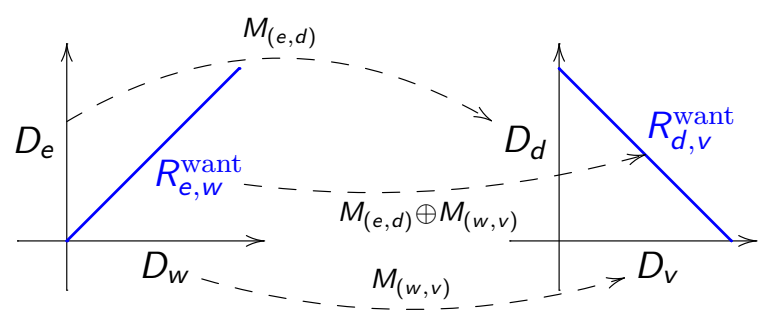

Initial correspondence: $R_{e, w}^{\text {want }}=\{(i, i) \mid 0 \leq i \leq N\}$

Propagation: $R_{d, v}^{\text {want }}=\left(M_{(e, d)} \oplus M_{(w, v)}\right) R_{e, w}^{\text {want }}$

Correspondence at input nodes holds for equal indices

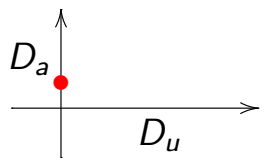

$$
\Rightarrow R^{\text {got }}=R^{\text {want }} \cap\{(i, i)\}
$$

$\Rightarrow R^{\text {lost }}=R^{\text {want }} \backslash\{(i, i)\}$

Backpropagation
- $R_{u_{1}, u_{2}}^{\text {lost }}=$

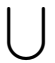
$\left(M_{\left(u_{1}, v_{1}\right)}^{-1} \oplus M_{\left(u_{2}, v_{2}\right)}^{-1}\right) R_{v_{1}, v_{2}}^{\text {lost }}$$$
\left(u_{1}, v_{1}\right),\left(u_{2}, v_{2}\right) \text { s.t. } p_{\left(u_{1}, v_{1}\right)}=p_{\left(u_{2}, v_{2}\right)}
$$

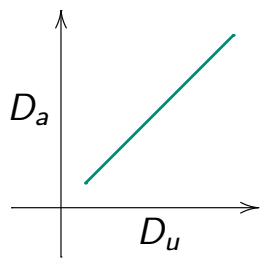

- $R^{\text {got }}=R^{\text {want }} \backslash R^{\text {lost }}$ 


\section{Propagation of Correspondences}
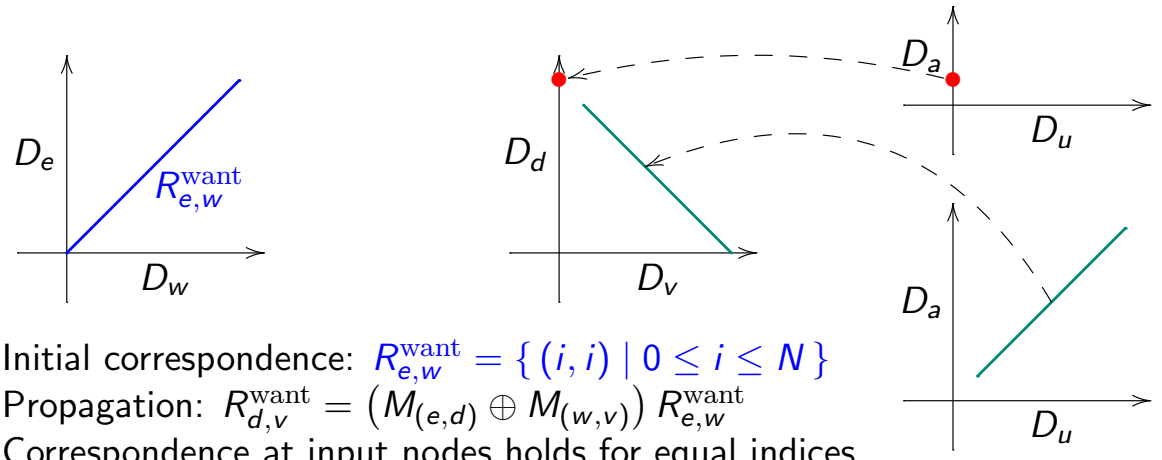

Initial correspondence: $R_{e, w}^{\text {want }}=\{(i, i) \mid 0 \leq i \leq N\}$

Propagation: $R_{d, v}^{\text {want }}=\left(M_{(e, d)} \oplus M_{(w, v)}\right) R_{e, w}^{\text {want }}$

Correspondence at input nodes holds for equal indices

$\Rightarrow R^{\text {got }}=R^{\text {want }} \cap\{(i, i)\}$

$\Rightarrow R^{\text {lost }}=R^{\text {want }} \backslash\{(i, i)\}$

Backpropagation

- $R_{u_{1}, u_{2}}^{\text {lost }}=$

$\left(M_{\left(u_{1}, v_{1}\right)}^{-1} \oplus M_{\left(u_{2}, v_{2}\right)}^{-1}\right) R_{v_{1}, v_{2}}^{\text {lost }}$

$\left(u_{1}, v_{1}\right),\left(u_{2}, v_{2}\right)$ s.t. $p_{\left(u_{1}, v_{1}\right)}=p_{\left(u_{2}, v_{2}\right)}$

- $R^{\text {got }}=R^{\text {want }} \backslash R^{\text {lost }}$ 


\section{Propagation of Correspondences}

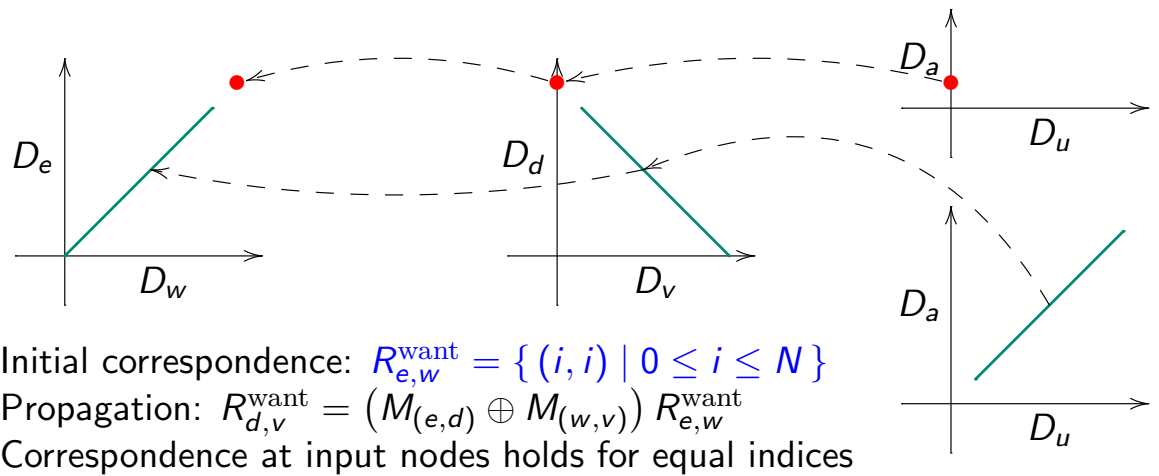

$\Rightarrow R^{\text {got }}=R^{\text {want }} \cap\{(i, i)\}$

$\Rightarrow R^{\text {lost }}=R^{\text {want }} \backslash\{(i, i)\}$

Backpropagation

- $R_{u_{1}, u_{2}}^{\text {lost }}=\bigcup_{\left(u_{1}, v_{1}\right),\left(u_{2}, v_{2}\right)}$ s.t. $p_{\left(u_{1}, v_{1}\right)}=p_{\left(u_{2}, v_{2}\right)}\left(M_{\left(u_{1}, v_{1}\right)}^{-1} \oplus M_{\left(u_{2}, v_{2}\right)}^{-1}\right) R_{v_{1}, v_{2}}^{\text {lost }}$

- $R^{\text {got }}=R^{\text {want }} \backslash R^{\text {lost }}$ 


\section{Propagation of Correspondences}

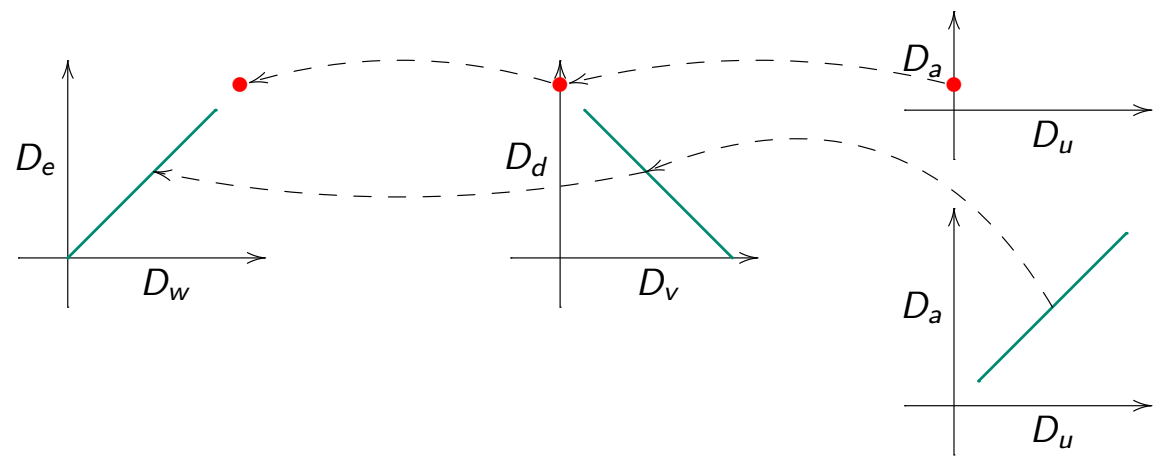

for $(i=0 ; i<=N ;++i)$ $\mathrm{a}[\mathrm{i}]=$ in $[i]$;

$\mathrm{a}[0]=$ in [1];

for $(i=0 ; i<=N ;++i)$ $b[i]=f(a[N-i])$;

for $(i=0 ; i<=N ;++i)$ Out (b[i]); for $(i=0 ; i<=N ;++i)\{$ $\mathrm{a}[\mathrm{i}]=$ in $[i]$; $b[i]=f(a[i])$

\}

for $(i=0 ; i<=N ;++i)$ Out (b $[N-i])$; 


\section{Propagation of Correspondences}

- Correspondence between computations $u_{1}$ and $u_{2}$ that perform the same operation

- Propagate to arguments

$$
R_{v_{1}, v_{2}}^{\text {want }}=\left(M_{\left(u_{1}, v_{1}\right)} \oplus M_{\left(u_{2}, v_{2}\right)}\right) R_{u_{1}, u_{2}}^{\text {want }}
$$

- Propagate results back

$$
R_{u_{1}, u_{2}}^{\text {lost }}=\underset{\left(u_{1}, v_{1}\right),\left(u_{2}, v_{2}\right)}{\bigcup_{\text {s.t. }} p_{\left(u_{1}, v_{1}\right)}=p_{\left(u_{2}, v_{2}\right)}}\left(M_{\left(u_{1}, v_{1}\right)}^{-1} \oplus M_{\left(u_{2}, v_{2}\right)}^{-1}\right) R_{v_{1}, v_{2}}^{\text {lost }}
$$




\section{Propagation of Correspondences}

- Correspondence between computations $u_{1}$ and $u_{2}$ that perform the same operation

- Propagate to arguments

$$
R_{v_{1}, v_{2}}^{\text {want }}=\left(M_{\left(u_{1}, v_{1}\right)} \oplus M_{\left(u_{2}, v_{2}\right)}\right) R_{u_{1}, u_{2}}^{\text {want }}
$$

- Propagate results back

$$
R_{u_{1}, u_{2}}^{\text {lost }}=\bigcup_{\left(u_{1}, v_{1}\right),\left(u_{2}, v_{2}\right)} \bigcup_{\text {s.t. } p_{\left(u_{1}, v_{1}\right)}=p_{\left(u_{2}, v_{2}\right)}}\left(M_{\left(u_{1}, v_{1}\right)}^{-1} \oplus M_{\left(u_{2}, v_{2}\right)}^{-1}\right) R_{v_{1}, v_{2}}^{\text {lost }}
$$

- Special case: constant functions

$$
R_{u_{1}, u_{2}}^{\operatorname{lost}}=\emptyset
$$




\section{Propagation of Correspondences}

- Correspondence between computations $u_{1}$ and $u_{2}$ that perform the same operation

- Propagate to arguments

$$
R_{v_{1}, v_{2}}^{\text {want }}=\left(M_{\left(u_{1}, v_{1}\right)} \oplus M_{\left(u_{2}, v_{2}\right)}\right) R_{u_{1}, u_{2}}^{\text {want }}
$$

- Propagate results back

$$
R_{u_{1}, u_{2}}^{\text {lost }}=\bigcup_{\left(u_{1}, v_{1}\right),\left(u_{2}, v_{2}\right)} \bigcup_{\text {s.t. } p_{\left(u_{1}, v_{1}\right)}=p_{\left(u_{2}, v_{2}\right)}}\left(M_{\left(u_{1}, v_{1}\right)}^{-1} \oplus M_{\left(u_{2}, v_{2}\right)}^{-1}\right) R_{v_{1}, v_{2}}^{\text {lost }}
$$

- Special case: constant functions

$$
R_{u_{1}, u_{2}}^{\operatorname{lost}}=\emptyset
$$

- Computations performing different operations

$$
R_{u_{1}, u_{2}}^{\text {lost }}=R_{u_{1}, u_{2}}^{\text {want }}
$$




\section{Propagation of Correspondences}

- Correspondence between computations $u_{1}$ and $u_{2}$ that perform the same operation

- Propagate to arguments

$$
R_{v_{1}, v_{2}}^{\text {want }}=\left(M_{\left(u_{1}, v_{1}\right)} \oplus M_{\left(u_{2}, v_{2}\right)}\right) R_{u_{1}, u_{2}}^{\text {want }}
$$

- Propagate results back

$$
R_{u_{1}, u_{2}}^{\text {lost }}=\bigcup_{\left(u_{1}, v_{1}\right),\left(u_{2}, v_{2}\right)} \bigcup_{\text {s.t. } p_{\left(u_{1}, v_{1}\right)}=p_{\left(u_{2}, v_{2}\right)}}\left(M_{\left(u_{1}, v_{1}\right)}^{-1} \oplus M_{\left(u_{2}, v_{2}\right)}^{-1}\right) R_{v_{1}, v_{2}}^{\text {lost }}
$$

- Special case: constant functions

$$
R_{u_{1}, u_{2}}^{\text {lost }}=\emptyset
$$

- Computations performing different operations

$$
R_{u_{1}, u_{2}}^{\text {lost }}=R_{u_{1}, u_{2}}^{\text {want }}
$$

- Input computations

$$
R_{u_{1}, u_{2}}^{\text {lost }}=R_{u_{1}, u_{2}}^{\text {want }} \backslash\{(\mathbf{i}, \mathbf{i})\}
$$




\section{Copy Propagation}

- Correspondence between computations $u_{1}$ and $u_{2}$ that perform the same operation

- Propagate to arguments

$$
R_{v_{1}, v_{2}}^{\text {want }}=\left(M_{\left(u_{1}, v_{1}\right)} \oplus M_{\left(u_{2}, v_{2}\right)}\right) R_{u_{1}, u_{2}}^{\text {want }}
$$

- Propagate results back

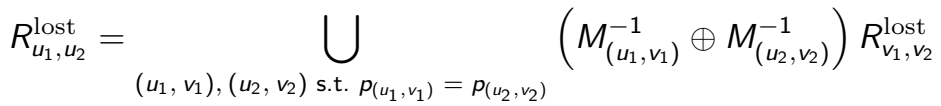




\section{Copy Propagation}

- Correspondence between computations $u_{1}$ and $u_{2}$ that perform the same operation

- Propagate to arguments

$$
R_{v_{1}, v_{2}}^{\text {want }}=\left(M_{\left(u_{1}, v_{1}\right)} \oplus M_{\left(u_{2}, v_{2}\right)}\right) R_{u_{1}, u_{2}}^{\text {want }}
$$

- Propagate results back

$$
R_{u_{1}, u_{2}}^{\text {lost }}=\bigcup_{\left(u_{1}, v_{1}\right),\left(u_{2}, v_{2}\right) \text { s.t. } p_{\left(u_{1}, v_{1}\right)}=p_{\left(u_{2}, v_{2}\right)}}\left(M_{\left(u_{1}, v_{1}\right)}^{-1} \oplus M_{\left(u_{2}, v_{2}\right)}^{-1}\right) R_{v_{1}, v_{2}}^{\text {lost }}
$$

- Copy propagation if $u_{1}$ performs "copy" operation (id)

- Propagate to argument

$$
R_{v_{1}, u_{2}}^{\text {want }}=\left(M_{\left(u_{1}, v_{1}\right)} \oplus 1_{D_{u_{2}}}\right) R_{u_{1}, u_{2}}^{\text {want }}
$$

- Propagate results back

$$
R_{u_{1}, u_{2}}^{\text {lost }}=\bigcup_{\left(u_{1}, v_{1}\right)}\left(M_{\left(u_{1}, v_{1}\right)}^{-1} \oplus 1_{D_{u_{2}}}\right) R_{v_{1}, u_{2}}^{\text {lost }}
$$




\section{Copy Propagation}

- Correspondence between computations $u_{1}$ and $u_{2}$ that perform the same operation

- Propagate to arguments

$$
R_{v_{1}, v_{2}}^{\text {want }}=\left(M_{\left(u_{1}, v_{1}\right)} \oplus M_{\left(u_{2}, v_{2}\right)}\right) R_{u_{1}, u_{2}}^{\text {want }}
$$

- Propagate results back

$$
R_{u_{1}, u_{2}}^{\text {lost }}=\bigcup_{\left(u_{1}, v_{1}\right),\left(u_{2}, v_{2}\right) \text { s.t. } p_{\left(u_{1}, v_{1}\right)}=p_{\left(u_{2}, v_{2}\right)}}\left(M_{\left(u_{1}, v_{1}\right)}^{-1} \oplus M_{\left(u_{2}, v_{2}\right)}^{-1}\right) R_{v_{1}, v_{2}}^{\text {lost }}
$$

- Copy propagation if $u_{1}$ performs "copy" operation (id)

- Propagate to argument

$$
R_{v_{1}, u_{2}}^{\text {want }}=\left(M_{\left(u_{1}, v_{1}\right)} \oplus 1_{D_{u_{2}}}\right) R_{u_{1}, u_{2}}^{\text {want }}
$$

- Propagate results back

$$
R_{u_{1}, u_{2}}^{\text {lost }}=\bigcup_{\left(u_{1}, v_{1}\right)}\left(M_{\left(u_{1}, v_{1}\right)}^{-1} \oplus 1_{D_{u_{2}}}\right) R_{v_{1}, u_{2}}^{\text {lost }}
$$

- Similarly if $u_{2}$ performs "copy" operation (id) 


\section{Commutative Operations}

- Correspondence between computations $u_{1}$ and $u_{2}$ that perform the same (non-commutative) operation

- Propagate to arguments

$$
R_{v_{1}, v_{2}}^{\text {want }}=\left(M_{\left(u_{1}, v_{1}\right)} \oplus M_{\left(u_{2}, v_{2}\right)}\right) R_{u_{1}, u_{2}}^{\text {want }}
$$

- Propagate results back

$$
R_{u_{1}, u_{2}}^{\text {lost }}=\bigcup_{\left(u_{1}, v_{1}\right),\left(u_{2}, v_{2}\right) \text { s.t. } p_{\left(u_{1}, v_{1}\right)}=p_{\left(u_{2}, v_{2}\right)}}\left(M_{\left(u_{1}, v_{1}\right)}^{-1} \oplus M_{\left(u_{2}, v_{2}\right)}^{-1}\right) R_{v_{1}, v_{2}}^{\text {lost }}
$$




\section{Commutative Operations}

- Correspondence between computations $u_{1}$ and $u_{2}$ that perform the same (non-commutative) operation

- Propagate to arguments

$$
R_{v_{1}, v_{2}}^{\text {want }}=\left(M_{\left(u_{1}, v_{1}\right)} \oplus M_{\left(u_{2}, v_{2}\right)}\right) R_{u_{1}, u_{2}}^{\text {want }}
$$

- Propagate results back

$$
R_{u_{1}, u_{2}}^{\text {lost }}=\bigcup_{\left(u_{1}, v_{1}\right),\left(u_{2}, v_{2}\right) \text { s.t. } p_{\left(u_{1}, v_{1}\right)}=p_{\left(u_{2}, v_{2}\right)}}\left(M_{\left(u_{1}, v_{1}\right)}^{-1} \oplus M_{\left(u_{2}, v_{2}\right)}^{-1}\right) R_{v_{1}, v_{2}}^{\text {lost }}
$$

- Correspondence between computations $u_{1}$ and $u_{2}$ that perform the same commutative operation

$\Rightarrow$ consider all permutations $\pi \in \Pi$ of the arguments 


\section{Commutative Operations}

- Correspondence between computations $u_{1}$ and $u_{2}$ that perform the same (non-commutative) operation

- Propagate to arguments

$$
R_{v_{1}, v_{2}}^{\text {want }}=\left(M_{\left(u_{1}, v_{1}\right)} \oplus M_{\left(u_{2}, v_{2}\right)}\right) R_{u_{1}, u_{2}}^{\text {want }}
$$

- Propagate results back

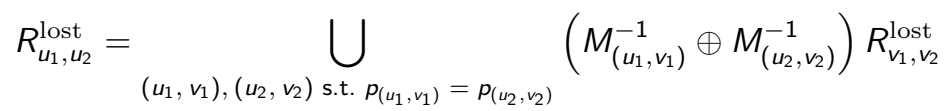

- Correspondence between computations $u_{1}$ and $u_{2}$ that perform the same commutative operation

$\Rightarrow$ consider all permutations $\pi \in \Pi$ of the arguments

- Propagate to arguments

$$
R_{v_{1}, v_{2}}^{\text {want }}=\left(M_{\left(u_{1}, v_{1}\right)} \oplus M_{\left(u_{2}, v_{2}\right)}\right) R_{u_{1}, u_{2}}^{\text {want }}
$$

- Propagate results back

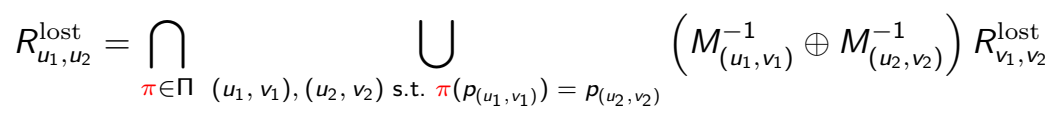




\section{Equivalence Checking Example}

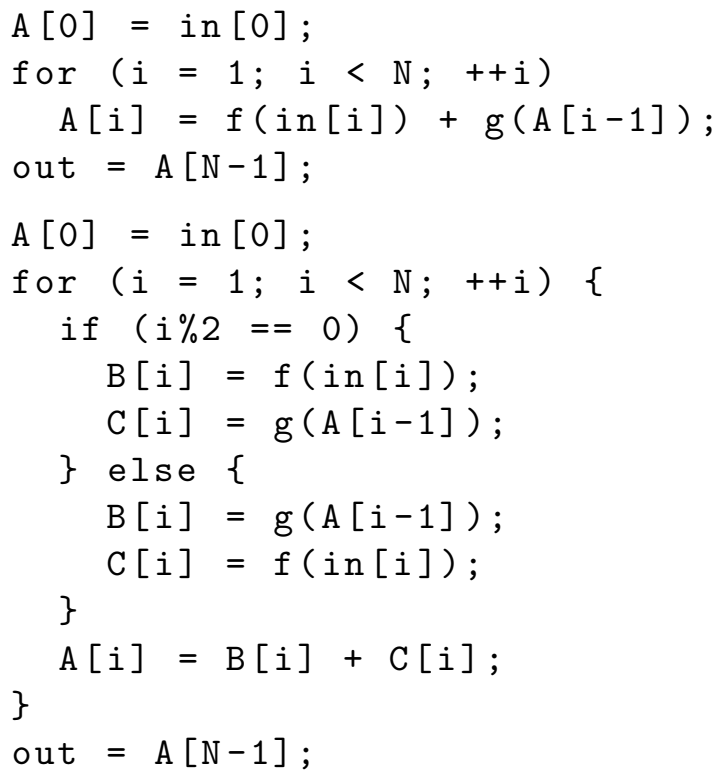

Are the two programs on the left equivalent?

$\Rightarrow$ Same output when given same input

Assumptions

- no pointers

- no recursion

- functions called are pure

- static control flow

- quasi-affine loop bounds

- quasi-affine conditions

- quasi-affine index expressions

Main challenges:

- Recurrences

- Transformations exploiting commutativity 


\section{Equivalence Checking Example}

$$
\begin{aligned}
& A[0]=\operatorname{in}[0] ; \\
& \text { for }(i=1 ; i<N ;++i)
\end{aligned}
$$$$
A[i]=f(i n[i])+g(A[i-1]) \text {; }
$$$$
\text { out }=\mathrm{A}[\mathrm{N}-1] \text {; }
$$$$
\mathrm{A}[0]=\text { in }[0] \text {; }
$$$$
\text { for }(i=1 ; i<N ;++i)\{
$$$$
\text { if }(i \% 2==0) \quad\{
$$$$
B[i]=f(\operatorname{in}[i]) \text {; }
$$$$
C[i]=g(A[i-1])
$$

\} else \{$$
B[i]=\$(A[i-1]) \text {; }
$$$$
C[i]=f(i n[i]) \text {; }
$$

\}

\}

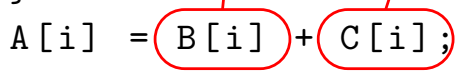

out $=\mathrm{A}[\mathrm{N}-1]$; 


\section{Equivalence Checking Example}

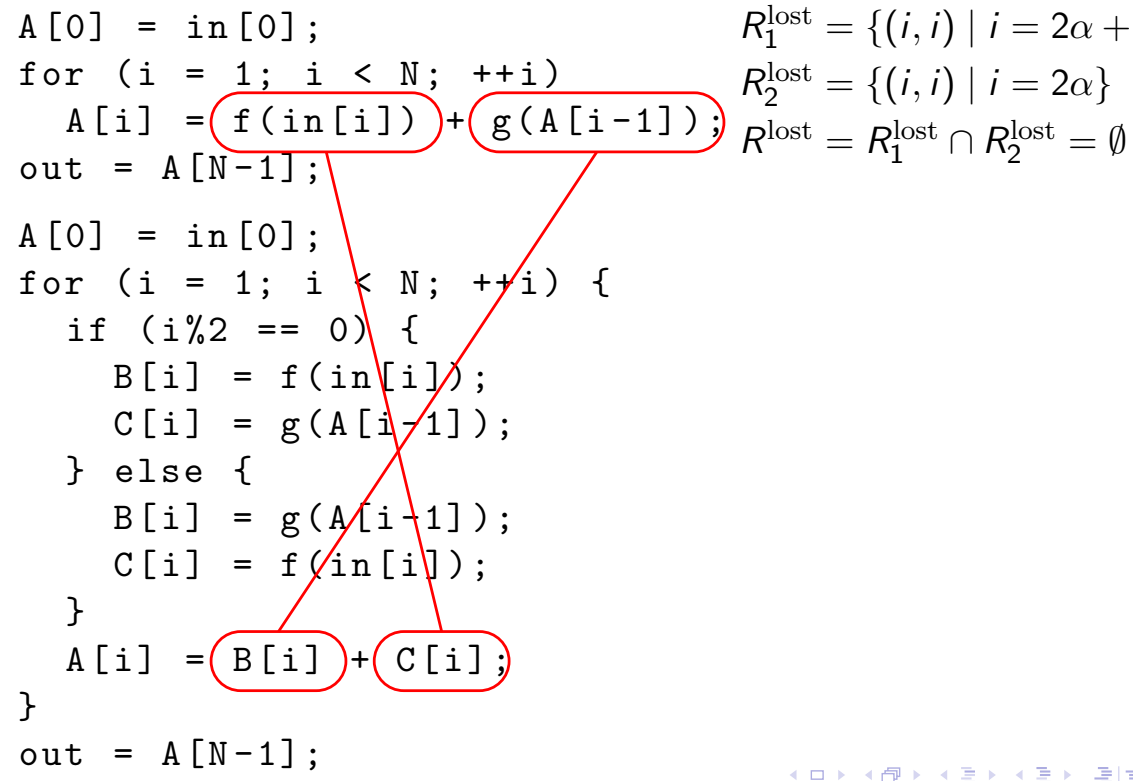




\section{Associative Operations}

Associative operations are "flattened" at the level of dependence graphs, prior to equivalence checking

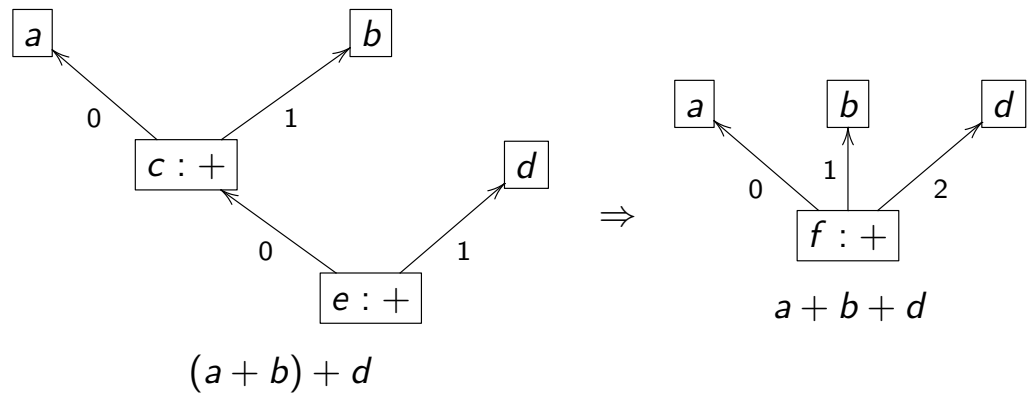

Dependence relations are adapted accordingly 


\section{Recurrences}

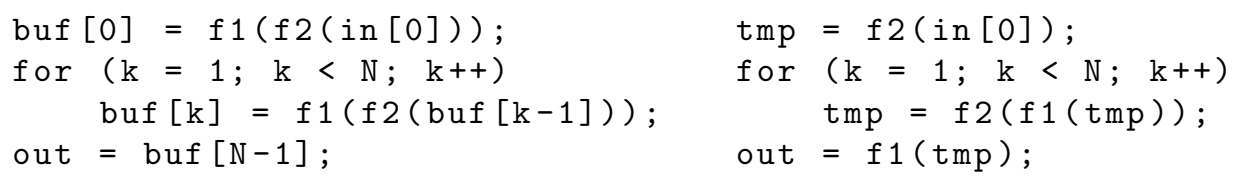




\section{Recurrences}

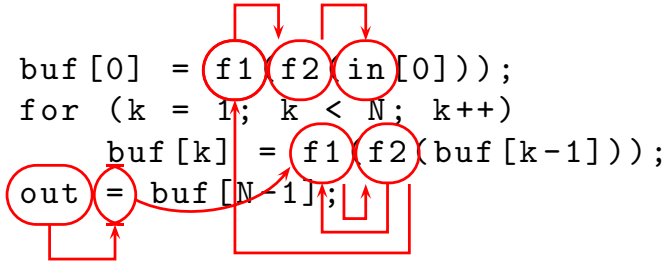

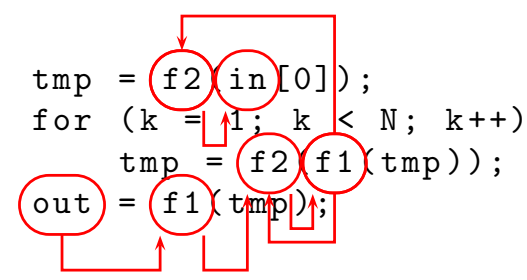




\section{Recurrences}

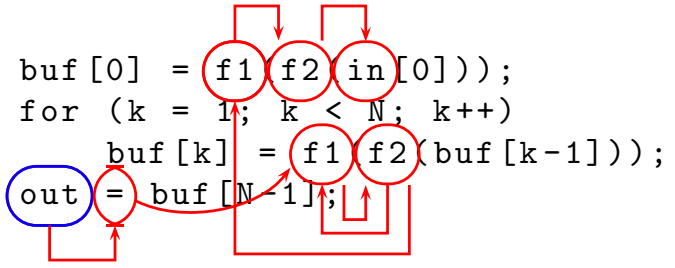

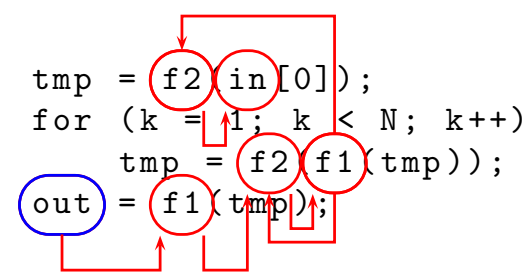

out 


\section{Recurrences}

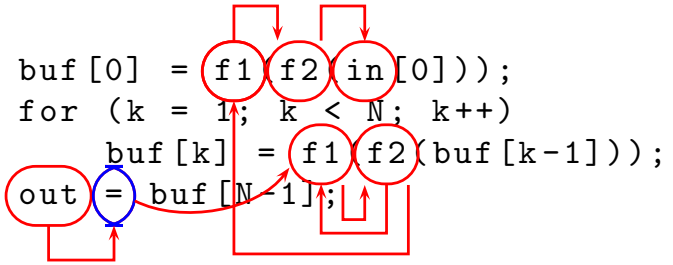

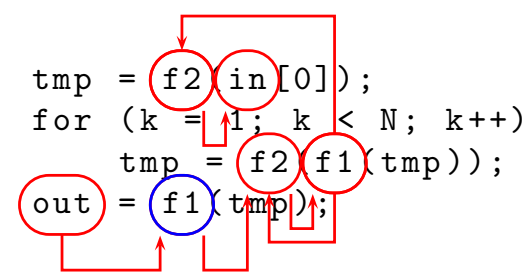

out $^{\bullet-} \rightarrow \operatorname{id} \underset{f 1}{\bullet}$ 


\section{Recurrences}

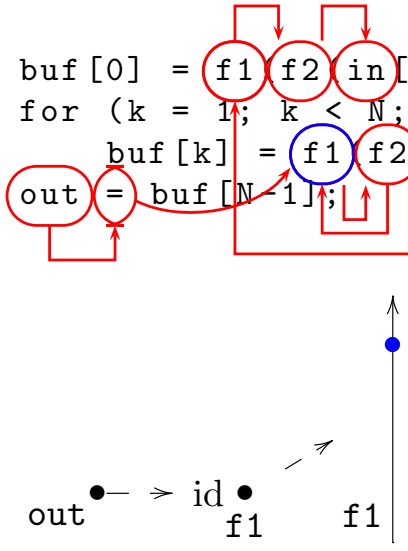

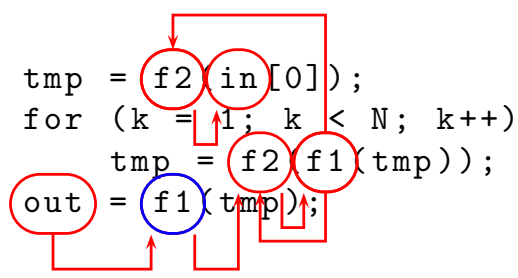

for $(\mathrm{k}=11 ; \mathrm{k}<\mathrm{N} ; \mathrm{k}++)$

out $=f(t \mathrm{tm})$ s: 


\section{Recurrences}

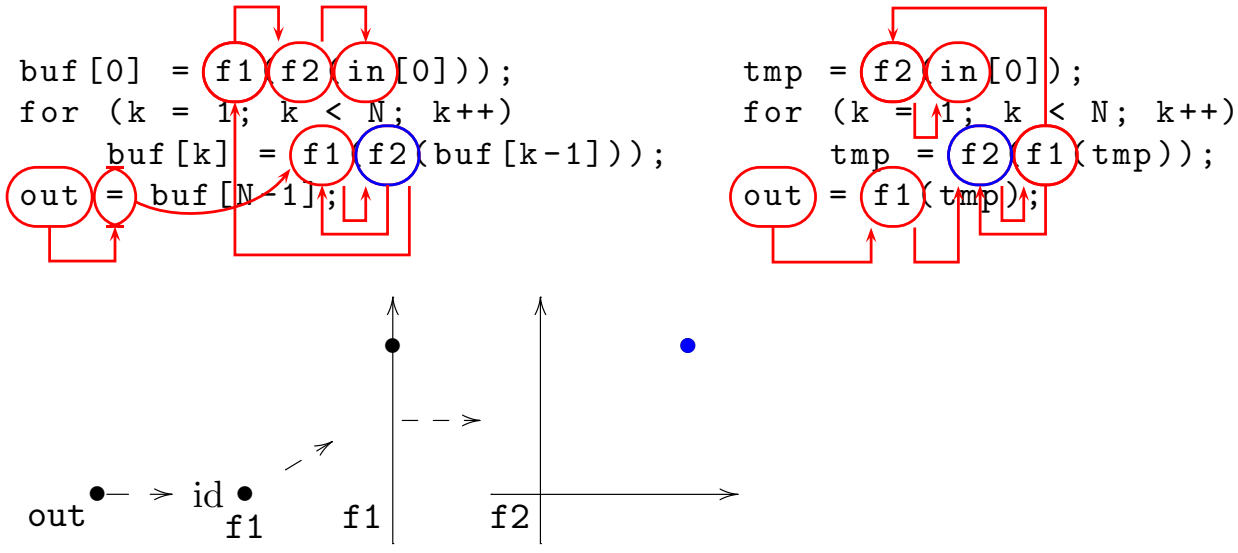




\section{Recurrences}

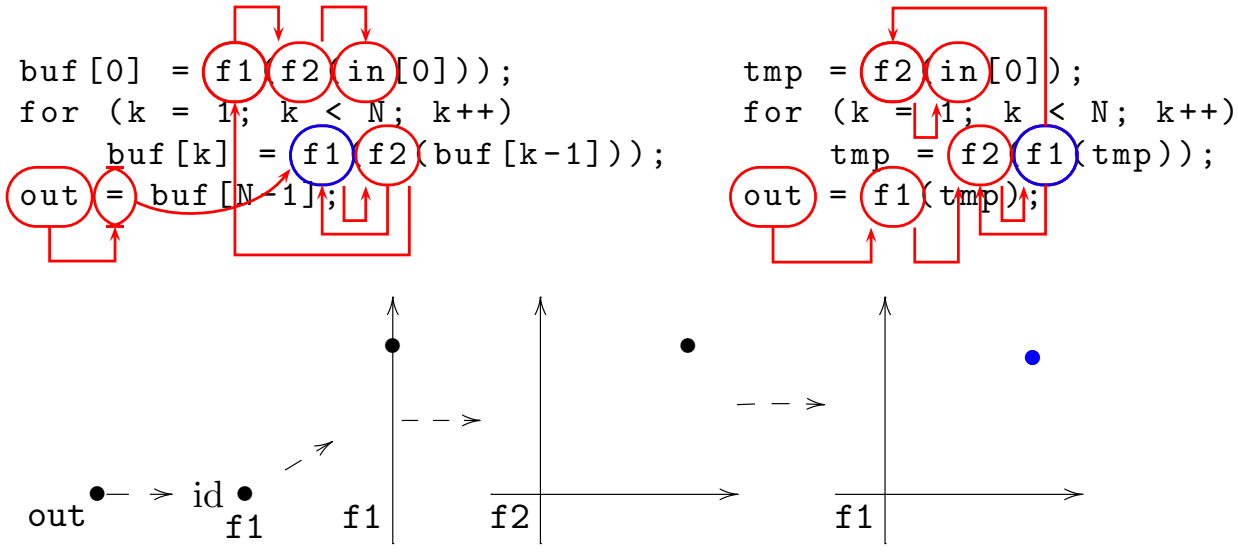




\section{Recurrences}

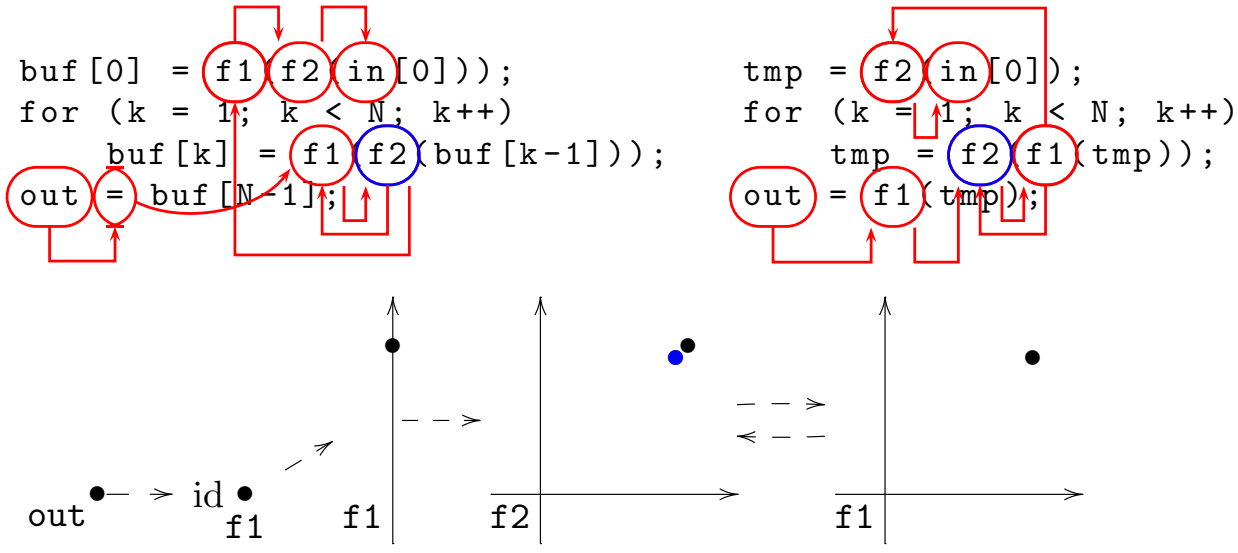




\section{Recurrences}

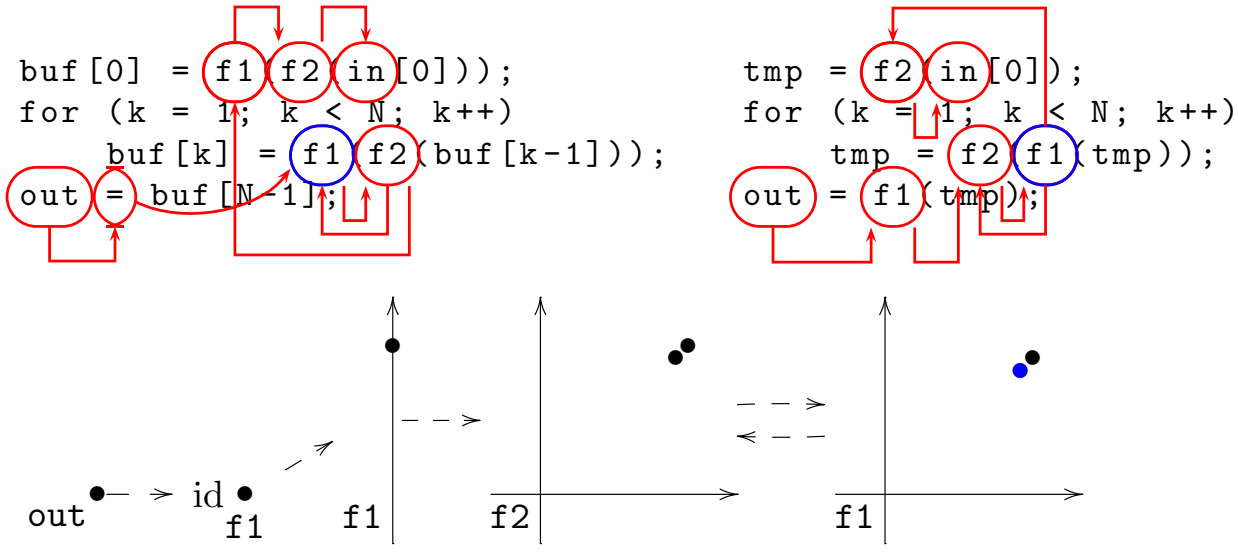




\section{Recurrences}

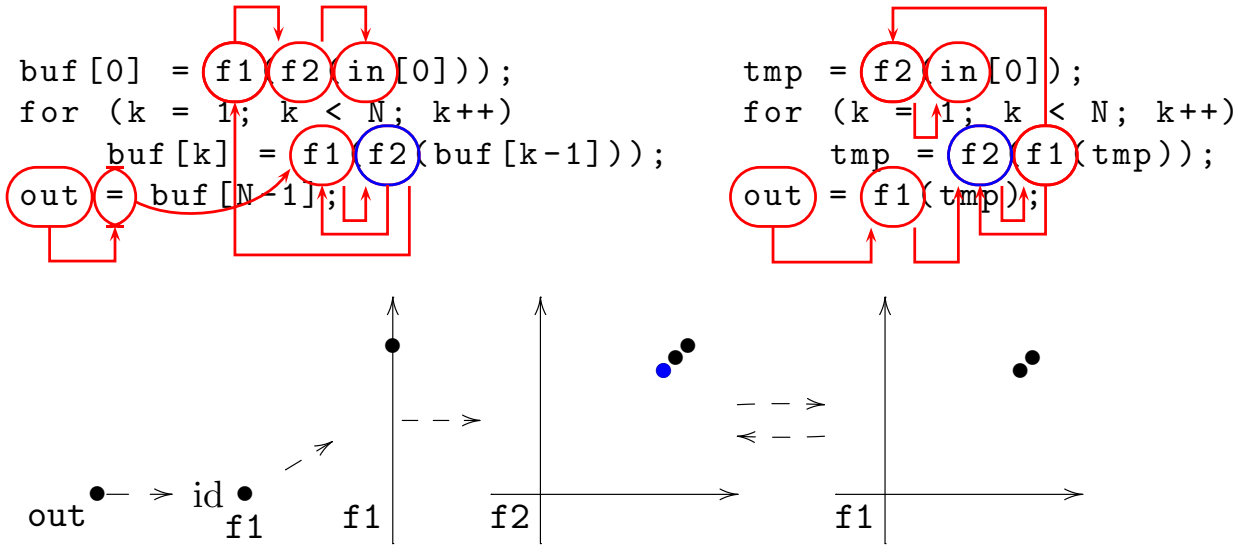




\section{Recurrences}

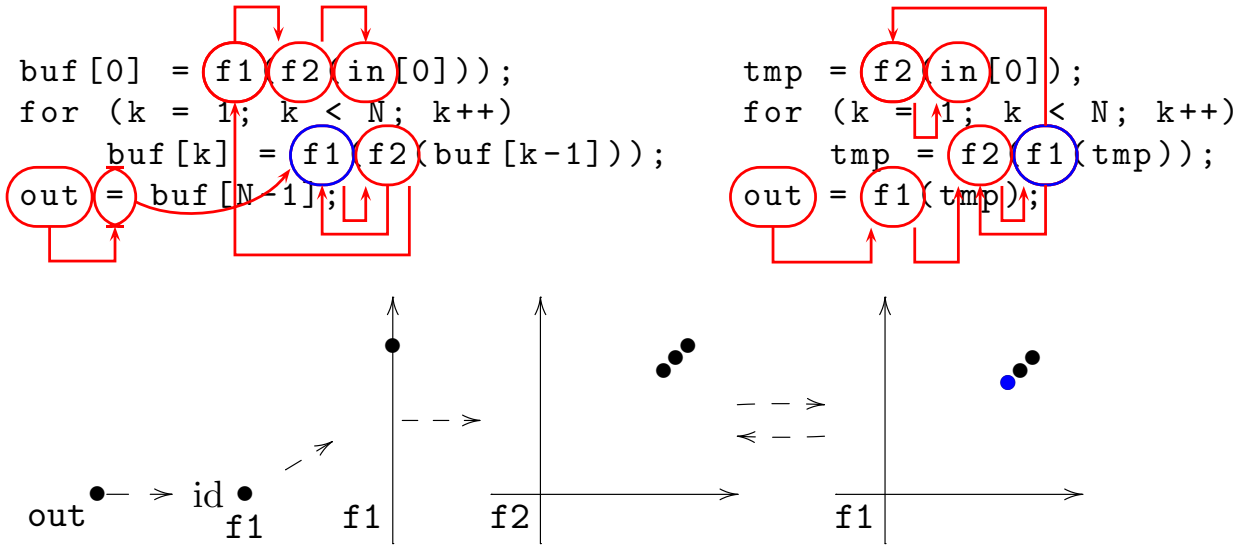




\section{Recurrences}

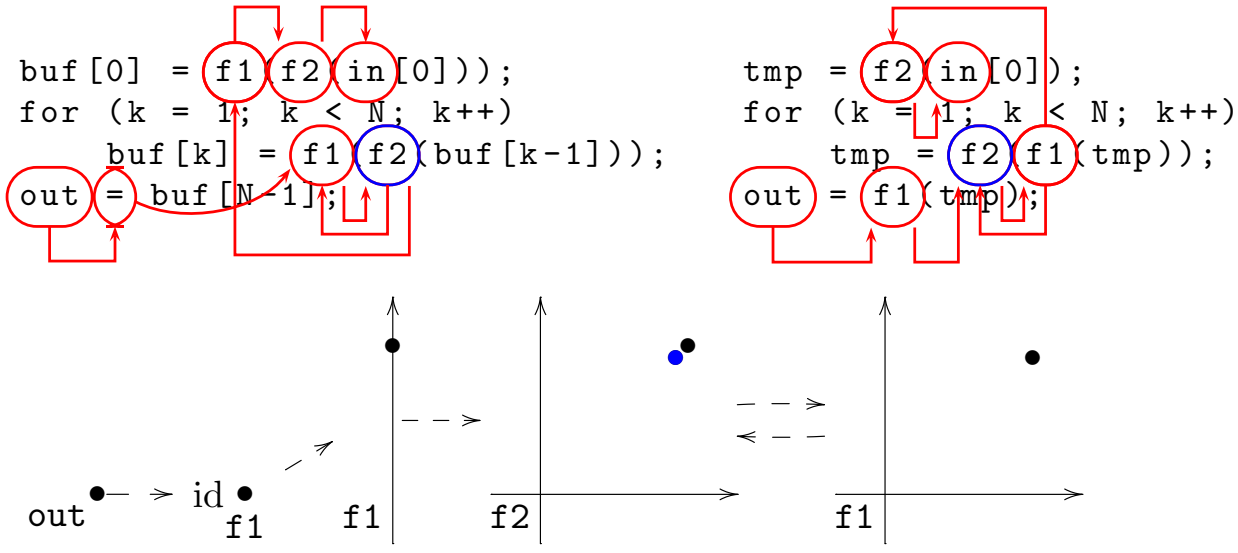




\section{Recurrences}

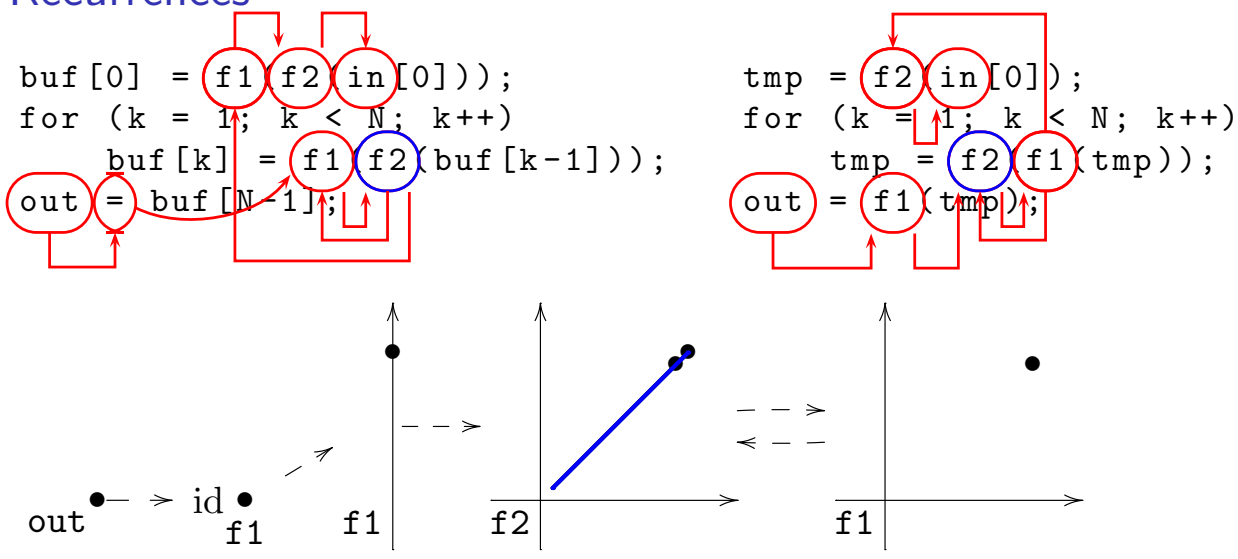

Apply widening: affine hull 


\section{Recurrences}

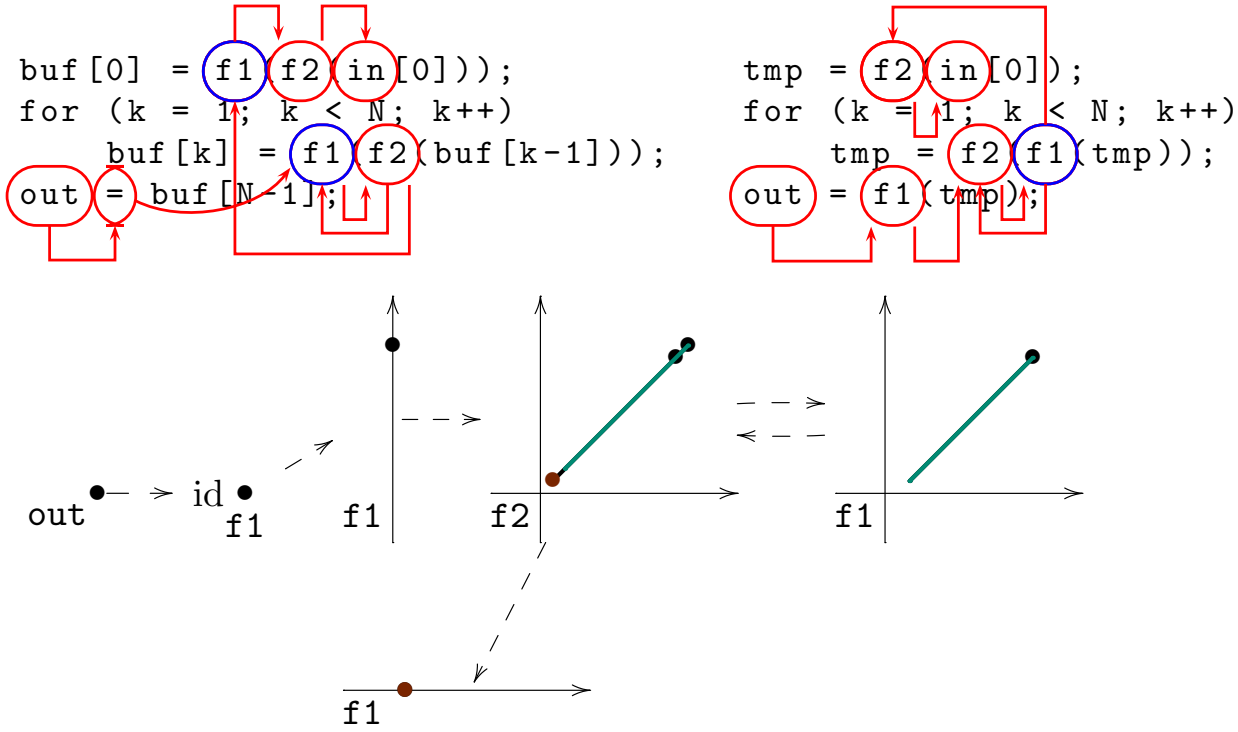




\section{Recurrences}

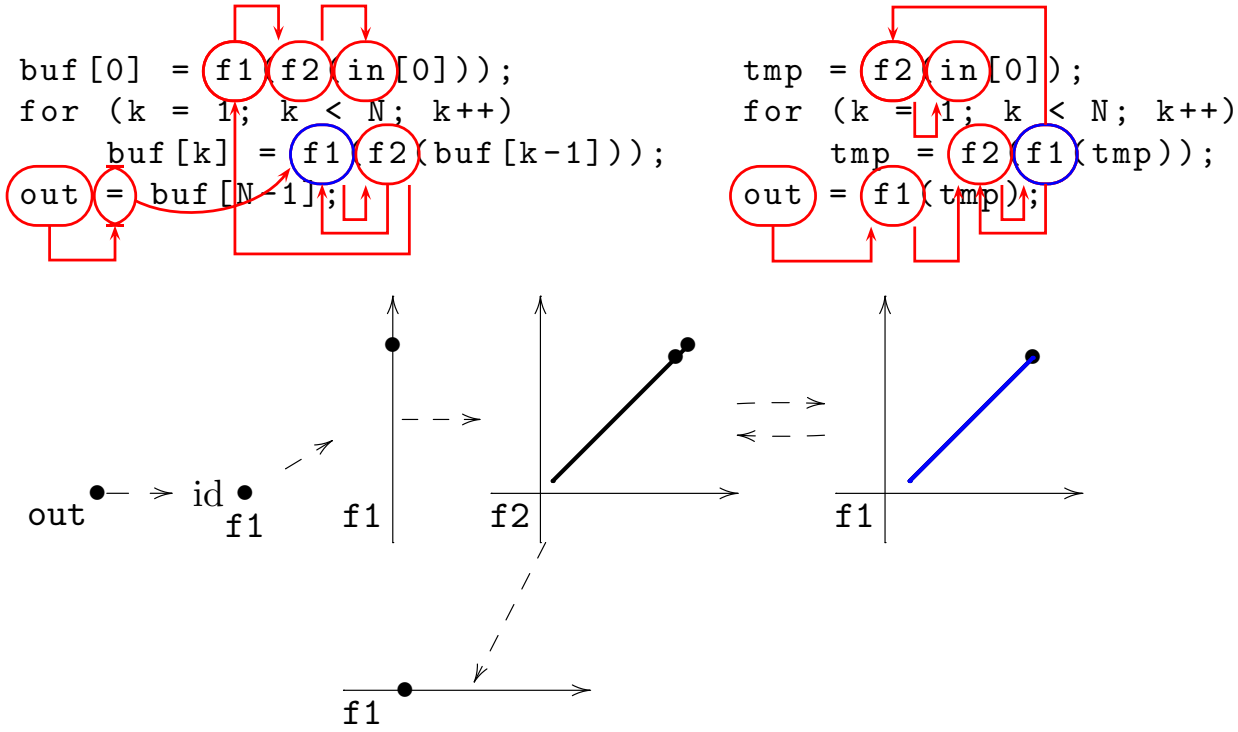




\section{Recurrences}

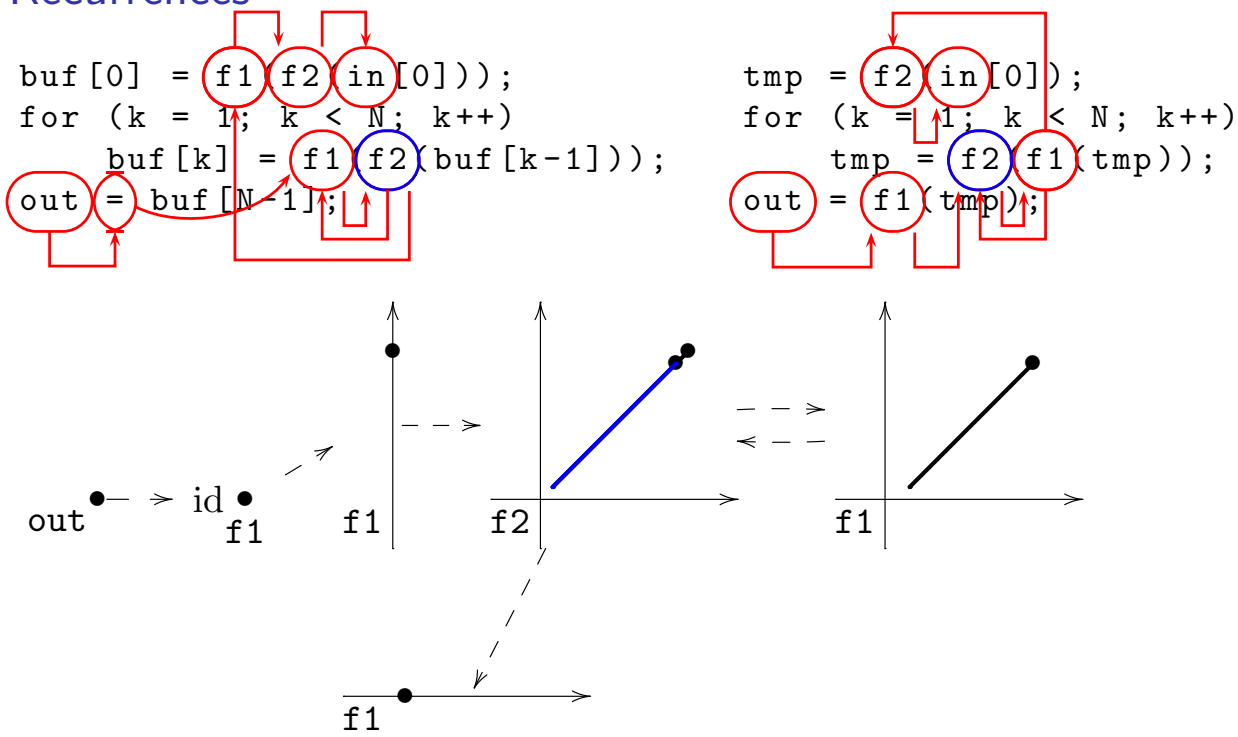




\section{Recurrences}

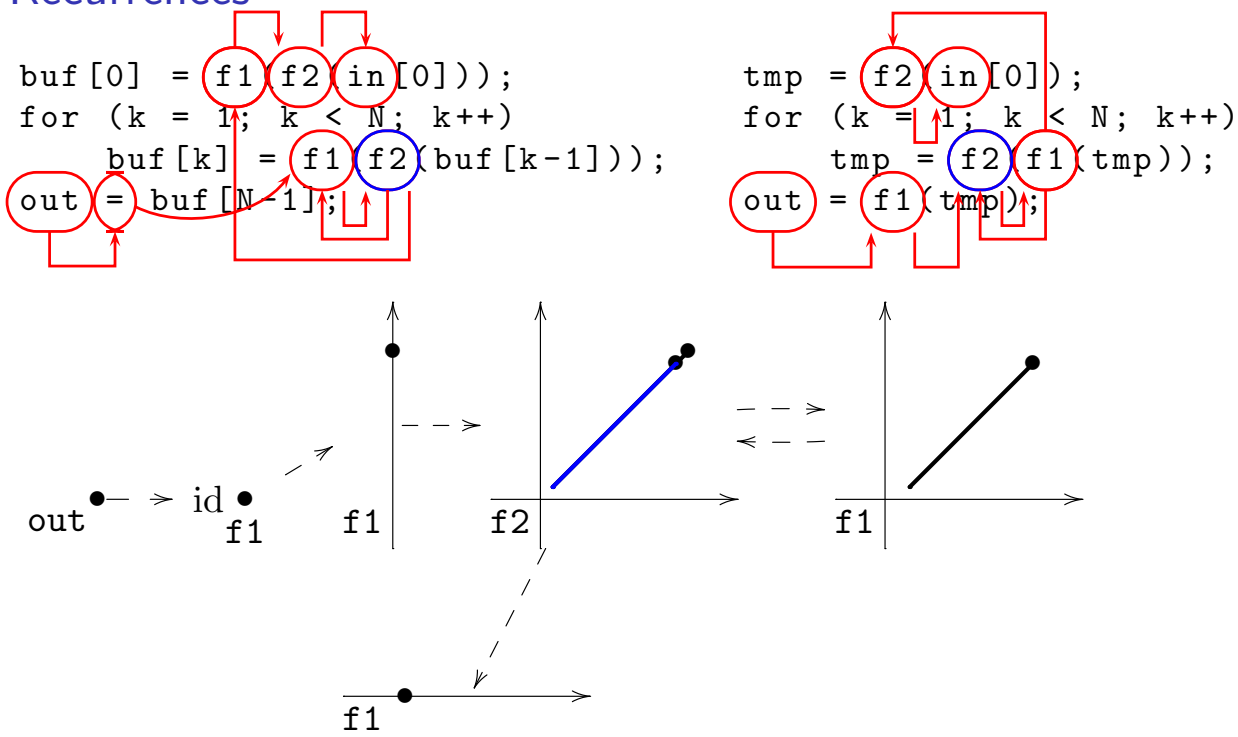

Apply induction 


\section{Recurrences}

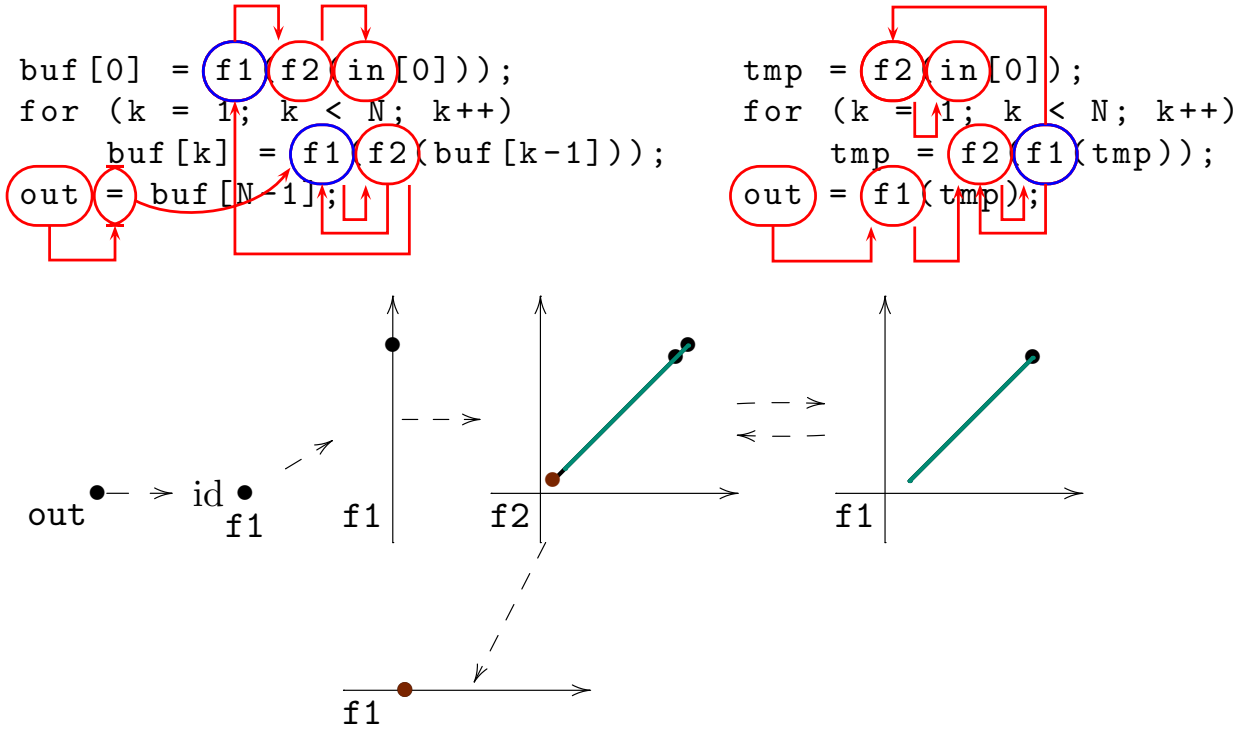




\section{Recurrences}

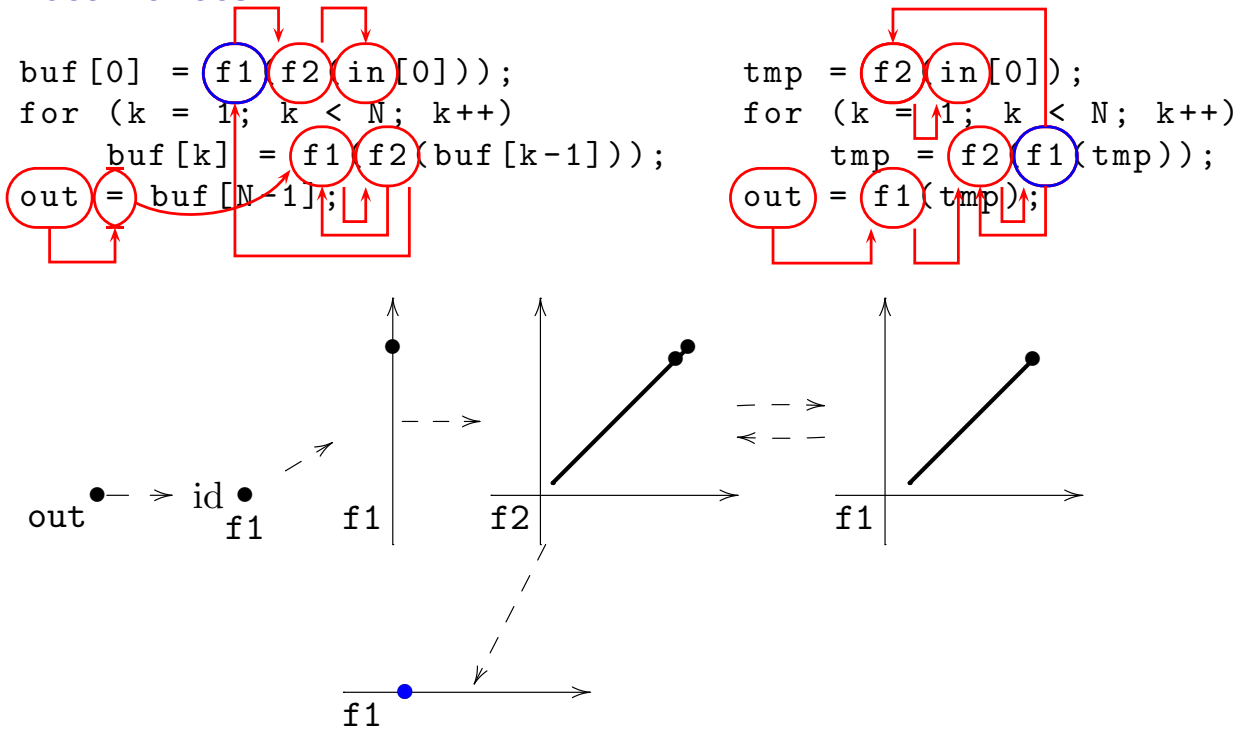




\section{Recurrences}

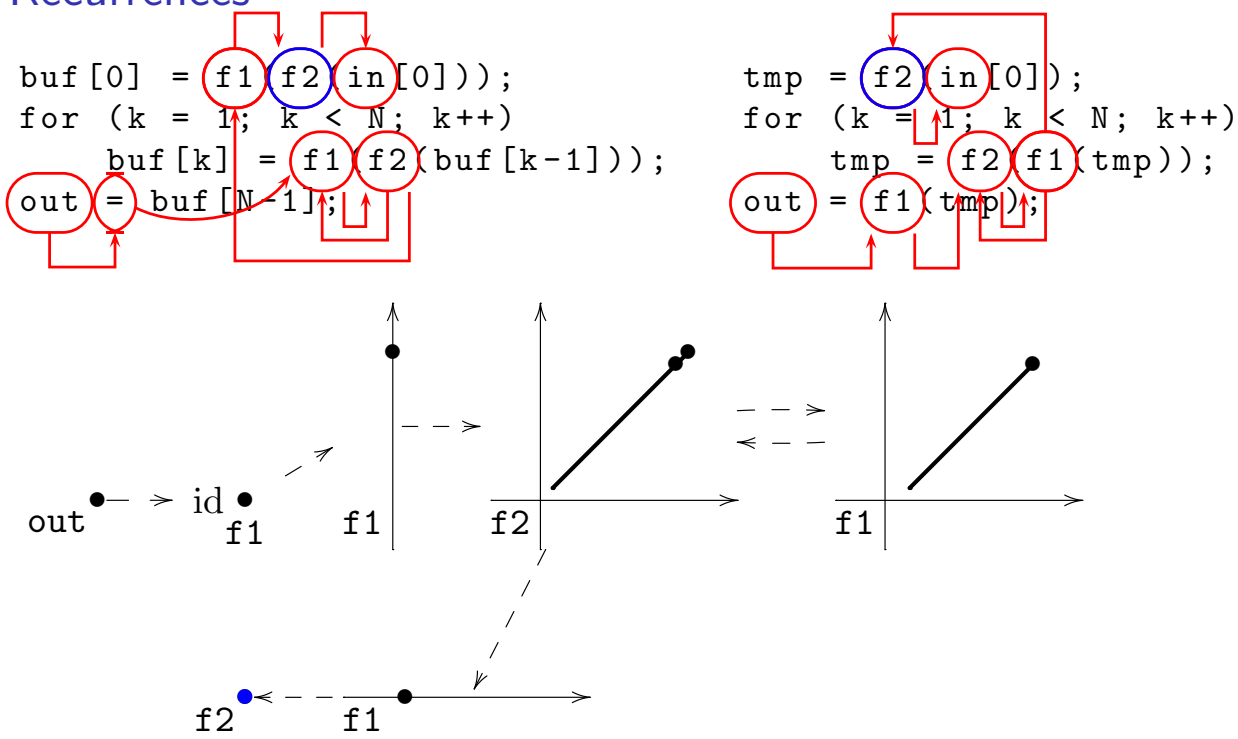




\section{Recurrences}

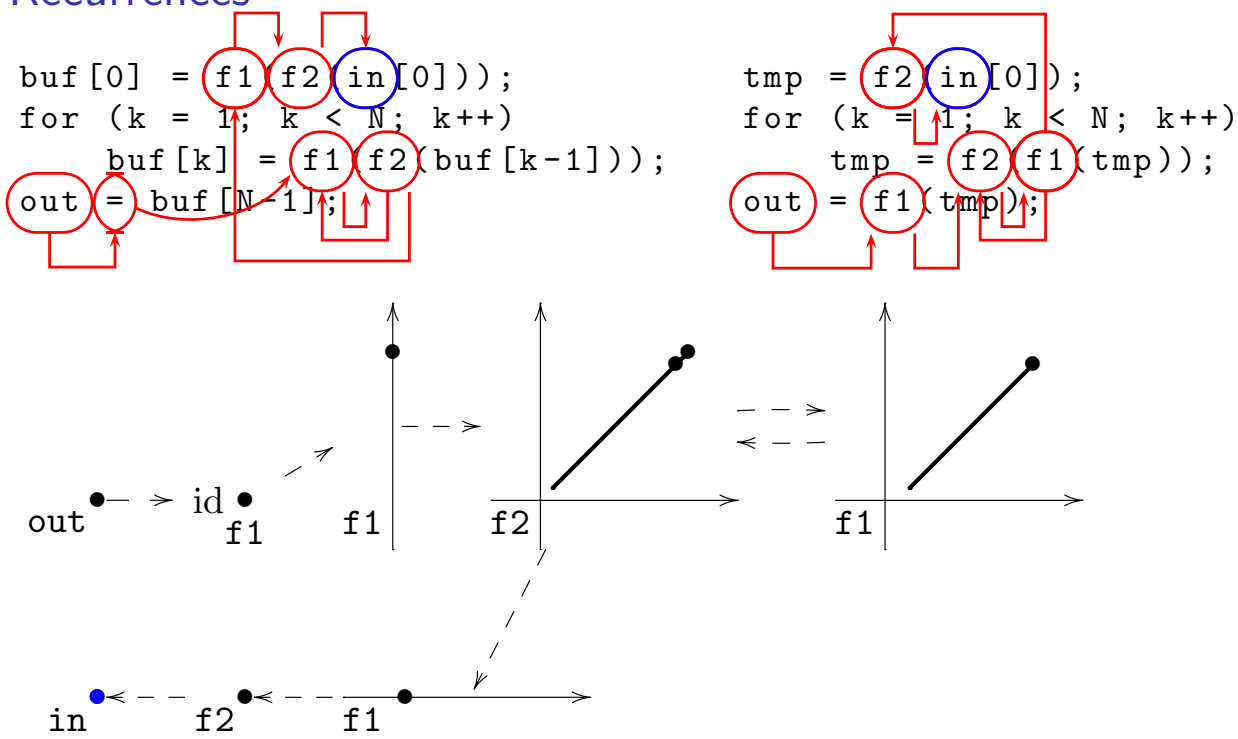




\section{Recurrences-Widening}

Sequence of $R^{\text {want }}=\{(N-1, N-1)\}$ and $R^{\text {want }}=\{(N-2, N-2)\}$ replaced by $R^{\text {want }}=\{(i, i) \mid 1 \leq i<N\}$ 


\section{Recurrences-Widening}

Sequence of $R^{\text {want }}=\{(N-1, N-1)\}$ and $R^{\text {want }}=\{(N-2, N-2)\}$ replaced by $R^{\text {want }}=\{(i, i) \mid 1 \leq i<N\}$

$\Rightarrow$ application of widening

$\Rightarrow$ turns ascending chain into eventually stationary ascending chain

$$
\begin{aligned}
& A_{0} \subseteq A_{1} \subseteq A_{2} \subseteq \cdots \\
& \text { In } \quad \cap \quad \text { In } \quad \exists N: \forall n \geq N: B_{n+1}=B_{n} \\
& B_{0} \subseteq B_{1} \subseteq B_{2} \subseteq \cdots
\end{aligned}
$$




\section{Recurrences-Widening}

Sequence of $R^{\text {want }}=\{(N-1, N-1)\}$ and $R^{\text {want }}=\{(N-2, N-2)\}$ replaced by $R^{\text {want }}=\{(i, i) \mid 1 \leq i<N\}$

$\Rightarrow$ application of widening

$\Rightarrow$ turns ascending chain into eventually stationary ascending chain

$$
\begin{aligned}
& A_{0} \subseteq A_{1} \subseteq A_{2} \subseteq \cdots \\
& \text { In } \quad \cap \quad \text { ก } \quad \exists N: \forall n \geq N: B_{n+1}=B_{n} \\
& B_{0} \subseteq B_{1} \subseteq B_{2} \subseteq \cdots
\end{aligned}
$$

Here: affine hull (smallest affine subspace containing $A_{i}$ )

$$
\begin{array}{ccc}
\{(N-1 ; N-1)\} & \subseteq\{(N-1 ; N-1),(N-2 ; N-2)\} \\
\{(N-1 ; N-1)\} \subseteq & \mid \cap \\
& \subseteq(i ; i)\}
\end{array}
$$




\section{Recurrences and Tabling}

Widening may be overestimate

$\Rightarrow$ important to keep track of unproved correspondence $R^{\text {lost }}$

Further occurrence of same pair of computations

$\Rightarrow$ further widening, or

$\Rightarrow$ apply induction hypothesis

$\Rightarrow$ after backpropagation of $R^{\text {lost }}$, check hypotheses do not intersect $R^{\text {lost }}$

$\Rightarrow$ perform "narrowing" if needed 


\section{Recurrences and Tabling}

Widening may be overestimate

$\Rightarrow$ important to keep track of unproved correspondence $R^{\text {lost }}$

Other occurrence of same pair of computations

$\Rightarrow$ widening, or

$\Rightarrow$ apply induction hypothesis

$\Rightarrow$ after backpropagation of $R^{\text {lost }}$, check hypotheses do not intersect $R^{\text {lost }}$

$\Rightarrow$ perform "narrowing" if needed

or

$\Rightarrow$ reuse tabled results $\left(R^{\text {lost }}\right)$ 


\section{Implementation Details}

All operations on sets/relations of integers performed by isl

- intersection

- union

- application of a map (relation) to a set, the domain of a relation or the range of a relation

- inverse of a relation (or application of an inverse map)

- integer affine hull (widening)

- subset testing

- equality testing

- emptiness testing 


\section{Implementation Details}

All operations on sets/relations of integers performed by isl

- intersection

- union

- application of a map (relation) to a set, the domain of a relation or the range of a relation

- inverse of a relation (or application of an inverse map)

- integer affine hull (widening)

- subset testing

- equality testing

- emptiness testing

Other features

- explicit support for existentially quantified variables

- uses PIP for solving (P)ILP problems

- all computations in exact integer arithmetic using GMP

- built-in incremental LP solver

- released under LGPL license 


\section{Experiments-CLooG}

Most experiments performed on output of CLooG

- CLooG generates code for scanning polyhedra

- Different backends (PolyLib/isl) may generate different code

- Experiment: prove different outputs are equivalent 


\section{Experiments-CLooG}

Most experiments performed on output of CLooG

- CLooG generates code for scanning polyhedra

- Different backends (PolyLib/isl) may generate different code

- Experiment: prove different outputs are equivalent

S1: $\{(i, j) \mid 1 \leq i \leq n \leq m \wedge j=i\}$

S2: $\{(i, j) \mid 1 \leq i \leq n \leq m \wedge i \leq j \leq n\}$

S3: $\{(i, j) \mid 1 \leq i \leq m \wedge j=n \leq m\}$
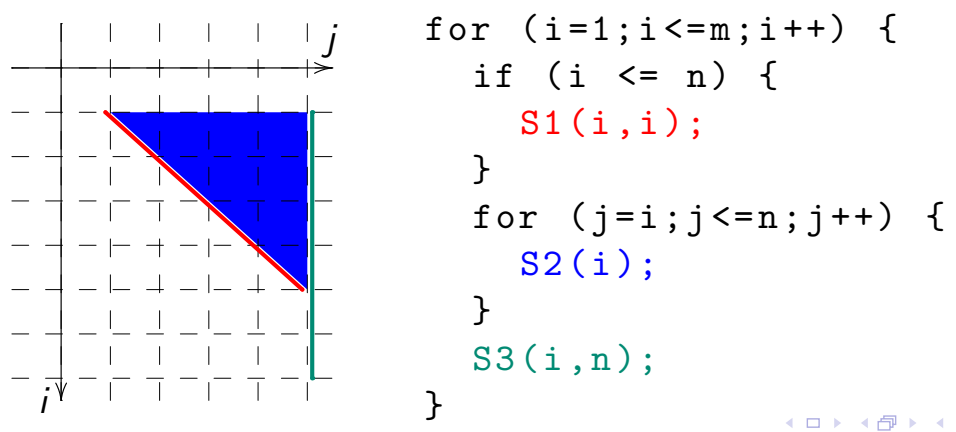


\section{CLooG Experiment Example}

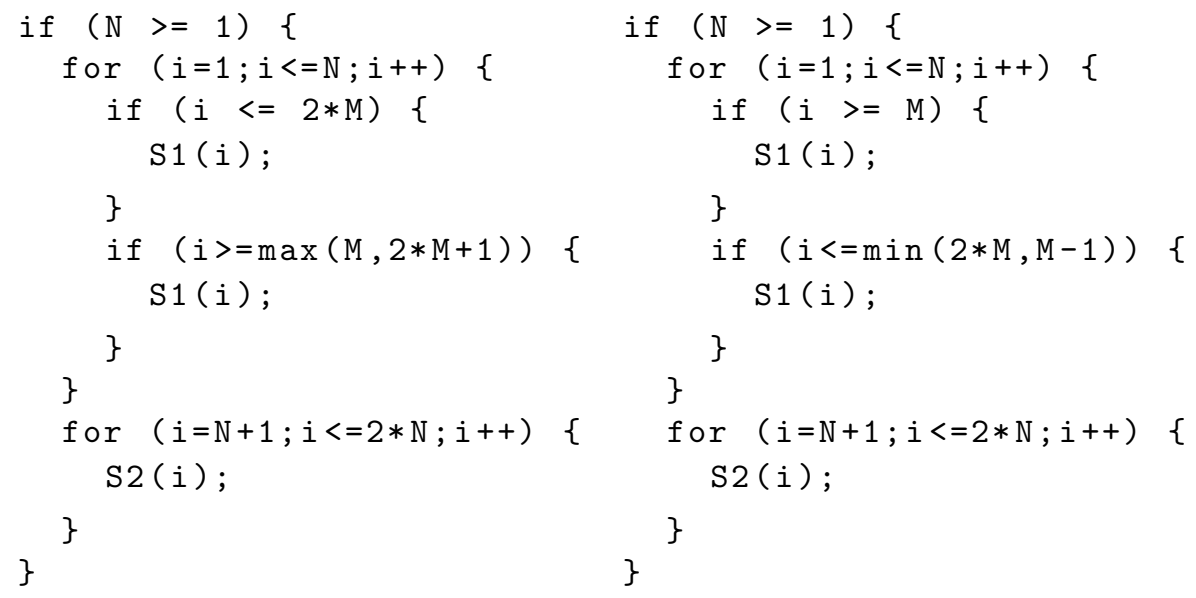




\section{CLooG Experiment Example}

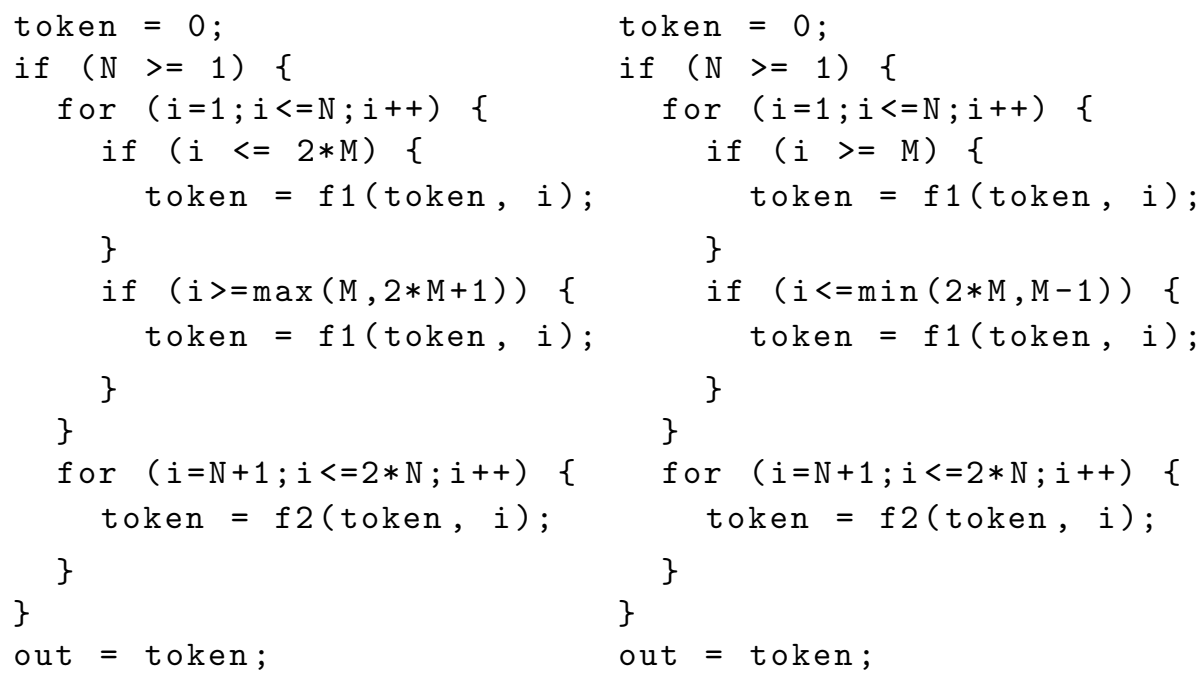




\section{Experimental Results}

\begin{tabular}{lrrrrr} 
Program pair & cases & lines & loops & time & widenings \\
\hline Motivating example & 1 & 47 & 2 & $0.03 \mathrm{~s}$ & 1 \\
USVD & 1 & 652 & 98 & $0.63 \mathrm{~s}$ & 0 \\
CLooG test 1 & 1 & 62 & 4 & $0.06 \mathrm{~s}$ & 4 \\
CLooG test 2 & 1 & 291 & 34 & $0.59 \mathrm{~s}$ & 51 \\
CLooG test 3 & 1 & 1634 & 108 & $19.85 \mathrm{~s}$ & 116 \\
CLooG test suite & 99 & 10804 & 1384 & $44.41 \mathrm{~s}$ & 1269
\end{tabular}




\section{Conclusions}

Novel approach for equivalence checking of static affine programs

- uses widening (affine hull) instead of transitive closure for handling recurrence

- supports non-uniform recurrences

- supports associative and commutative operations with fixed number of arguments

- publicly available prototype implementation (http://www.kotnet.org/ skimo/loop/isa-0.08.tar.bz2)

Future work

- associative operations with a variable number of arguments, e.g., different encodings of $\sum_{0 \leq i<N} \mathrm{a}[i]$

- general reductions

- data-dependent and non-affine conditions and index expressions 


\section{Partial Loop Unrolling}

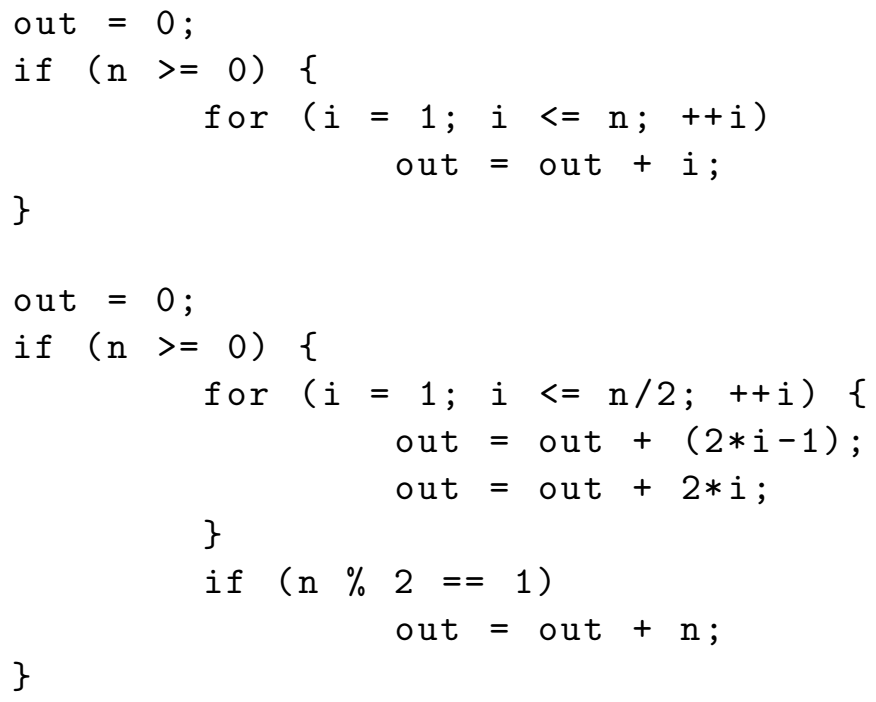

